\title{
Quasiperiodic functions theory and the superlattice potentials for a two-dimensional electron gas.
}

\author{
Andrei Ya. Maltsev* \\ L.D.Landau Institute for Theoretical Physics, 117940 ul. Kosygina 2, Moscow
}

(Dated: May 4, 2021)

\begin{abstract}
We consider Novikov problem of the classification of level curves of quasiperiodic functions on the plane and its connection with the conductivity of two-dimensional electron gas in the presence of both orthogonal magnetic field and the superlattice potentials of special type. We show that the modulation techniques used in the recent papers on the 2D heterostructures permit to obtain general quasiperiodic potentials for $2 \mathrm{D}$ electron gas and consider the asymptotic limit of conductivity when $\tau \rightarrow \infty$. Using the theory of quasiperiodic functions we introduce here the topological characteristics of such potentials observable in the conductivity. The corresponding characteristics are the direct analog of the "topological numbers" introduced previously in the conductivity of normal metals.

PACS numbers: 02.40.-k, 05.45.-a, 72.20My
\end{abstract}

\section{INTRODUCTION.}

In the present paper we consider modern experimental techniques of potential modulation for the twodimensional electron gas and show that they permit to obtain quasiperiodic potentials on the plane with different numbers of quasiperiods. Then we use topological results concerning the geometry of the level curves of such potentials (S.P. Novikov problem) to obtain the asymptotic $(\tau \rightarrow \infty)$ behavior of the conductivity phenomena in these systems. Namely, we consider the quasiclassical approach, where the quasiclassical cyclotron orbits drift along the level curves of potential in the presence of magnetic field $B$ which makes the geometry of such level curves important for transport phenomena. Our approach is based on the topological methods used previously (by S.P. Novikov and the author) in the theory of normal metals $(15,21,26)$ and the quasiclassical description of the transport phenomena in high-mobility 2D electron gas introduced by C.W.J. Beenakker $\left({ }^{30}\right)$ for the explanation of new oscillations in $B$-dependence of conductivity found in the periodically modulated $2 \mathrm{D}$ electron gas $\left(\frac{27}{)}\right)$.

We will consider here the cases of potentials with 3 and 4 quasiperiods and use a set of rather deep topological theorems concerning S.P. Novikov problem obtained during the last years. Let us say here that these two cases are actually the only cases which were studied seriously in topology and where very nice results were obtained. Namely, the full classification of the non-closed level curves was obtained for the case of potentials with 3 quasiperiods on the plane and it was shown (11,22) that only the so-called "topologically regular" level curves appear in the case when the non-closed level curves exist in a non-zero energy interval $\epsilon_{1} \leq V(\mathbf{r}) \leq \epsilon_{2}$. The corresponding curves reveal nice geometrical properties being bounded by straight strips of a finite width in the plane and passing through them. Moreover, it can be shown that the mean directions of these strips always correspond to some topological numbers characterizing the potential $V(\mathbf{r})$. Thus for the case of 3 quasiperiods these numbers can be represented as the indivisible inte- ger triples $\left(m_{1}, m_{2}, m_{3}\right)$ which can be defined experimentally from the mean directions of potential level curves. For the case of 4 quasiperiods the corresponding numbers are 4-tuples which can again be defined from the mean directions of the topologically regular open level curves in the transport phenomena. However, in the last case the existence of topologically regular open level curves can be stated only for small perturbations of purely periodic potentials in $\mathbb{R}^{2}(\stackrel{23}{)}$.

In this paper we show that special modulations of $2 \mathrm{D}$ electron gas give quasiperiodic potentials on the plane and introduce the corresponding topological numbers and their connection with the modulation pictures. Let us say that the topological numbers of this kind were introduced already in the theory of normal metals $(15,21,26)$ where the "geometric strong magnetic field limit" in the galvanomagnetic phenomena was considered. For this case only the situation with 3 quasiperiods was important and the topological numbers had the form of integer triples $\left(m_{1}, m_{2}, m_{3}\right)$. Another feature of the situation in the normal metals is that just the Fermi energy level $\epsilon_{F}$ is important for the asymptotic behavior of conductivity in the "geometric limit".

As we already said, we will use here the "drifting orbits" approximation and consider the case $\tau \rightarrow \infty$ which corresponds to the "geometric limit" in the situation of $2 \mathrm{D}$ electron gas. We consider in details the electric conductivity tensor $\sigma^{i k}$ in the asymptotic form for $\tau \rightarrow \infty$ when a strong anisotropy of $\sigma^{i k}$ reveals the mean directions of topologically regular trajectories and gives the corresponding topological numbers.

Let us say also that the cases of chaotic behavior of the potential level curves are also possible for the quasiperiodic potentials $V(\mathbf{r})(12,18)$. The asymptotic behavior of $\sigma^{i k}$ is more complicated in this case and we will not consider it here in details. For the case of 3 quasiperiods, however, the generic behavior of conductivity should correspond to topologically regular situation and the chaotic cases are "exclusive" unlike the cases with big numbers of quasiperiods. 


\section{BASIC DEFINITIONS AND HISTORICAL NOTES.}

According to the standard definition a quasiperiodic function $f(\mathbf{r}), \mathbf{r} \in \mathbb{R}^{n}$ with $N$ quasiperiods $(N \geq n)$ is a restriction of a periodic function $F(\mathbf{R}), \mathbf{R} \in \mathbb{R}^{N}$ with $N$ linearly independent periods $\mathbf{l}_{1}, \ldots, \mathbf{l}_{N}$ in a bigger linear space $\mathbb{R}^{N}$ to some "plane" $\mathbb{R}^{n} \subset \mathbb{R}^{N}$. The corresponding subspace $\mathbb{R}^{n}$ can then be given by a linear system

$$
\left\{\begin{array}{c}
a_{11} y^{1}+a_{12} y^{2}+\cdots+a_{1 N} y^{N}=b_{1} \\
\cdots \\
a_{N-n, 1} y^{1}+a_{N-n, 2} y^{2}+\cdots+a_{N-n, N} y^{N}=b_{N-n}
\end{array}\right.
$$

We will say that the plane $\mathbb{R}^{n}$ has the maximal irrationality if it is not parallel to any vector $\mathbf{l}$ belonging to the lattice $L$ generated by vectors $\mathbf{l}_{1}, \ldots, \mathbf{l}_{N}$ :

$$
L=\left\{p_{1} \mathbf{l}_{1}+\cdots+p_{N} \mathbf{l}_{N}, \quad p_{1}, \ldots, p_{N} \in \mathbb{Z}\right\}
$$

We will call the plane $\mathbb{R}^{n} \subset \mathbb{R}^{N}$ rational if it contains (i.e. parallel to) exactly $n$ linearly independent vectors belonging to $L$.

Obviously the generic planes $\mathbb{R}^{n}$ in $\mathbb{R}^{N}$ have the maximal irrationality. It is easy to see also that any vector $1 \in L$ parallel to the plane $\mathbb{R}^{n}$ in a non-generic situation becomes a period of the function $f(\mathbf{r})$ in $\mathbb{R}^{n}$. The function $f(\mathbf{r})$ corresponding to a rational plane $\mathbb{R}^{n} \subset \mathbb{R}^{N}$ is a $n$-periodic function in ordinary sense. It is easy to see also that generic quasiperiodic function $f(\mathbf{r})$ with $N$ quasiperiods has no periods in $\mathbb{R}^{n}$ for $N>n$.

We are going to consider the case $n=2$ such that the function $f(\mathbf{r})=f(x, y)$ is a quasiperiodic function on the two-dimensional plane $\mathbb{R}^{2}$. Namely, we will describe here important features of the global geometry of the level curves $f(\mathbf{r})=$ const (Novikov problem) which will play the main role for the phenomena discussed in this paper.

Let us say here that the Novikov problem is still unsolved for the case of arbitrary $N>2$ and we are going to deal here with the cases $N=3$ and $N=4$ where new topological and physical results were obtained during the last years $\left(\sec ^{\underline{\underline{6}}-\underline{26}}\right)$. According to the definition, the corresponding functions $f(\mathbf{r})$ will be the restrictions of periodic functions in $\mathbb{R}^{3}$ and $\mathbb{R}^{4}$ on some two-dimensional planes $\mathbb{R}^{2}$. Let us say here some words about this situation.

We will start with the very important case $N=3$ where the full classification of the curves $f(\mathbf{r})=$ const is constructed now. This case plays extremely important role for the galvanomagnetic phenomena in normal metals (see $15,21,26$ ) where the function $\hat{f}(\mathbf{p}), \mathbf{p}=\left(p_{1}, p_{2}, p_{3}\right)$ is defined in the space of quasimomenta of the Bloch electron in the crystal. The function $f(\mathbf{p})$ is a restriction of the three-periodic function $\hat{f}(\mathbf{p})$ on a two-dimensional plane (orthogonal to the magnetic field) embedded in $\mathbb{R}^{3}$. The level curves of $f(\mathbf{p})$ are the intersections of the corresponding plane $\Pi=\mathbb{R}^{2}$ with the 3 -periodic twodimensional level surfaces of the (smooth) function $\hat{f}(\mathbf{p})$ (dispersion relation). From the physical point of view the level curves of the function $f$ are the quasiclassical electron trajectories in the $\mathbf{p}$-space in the presence of magnetic field $\mathbf{B}$. We have in this case a one-parametric family of planes $\Pi$ orthogonal to $\mathbf{B}$ and a one-parametric set of the quasiperiodic functions defined in the different planes. The form of trajectory in the coordinate space is defined in this case by it's form in the p-space keeping all the main features of global geometry. For instance the projection of orbit on $x y$-plane in $\mathbf{r}$-space coincides precisely with the trajectory in $\mathbf{p}$-space rotated by $\pi / 2$. Let us also point out that only the trajectories close to the Fermi level are important for the case of normal metals.

The importance of the geometry of these trajectories for the galvanomagnetic phenomena was pointed out in ${ }^{1}-\underline{\underline{3}}$ (see also survey articles ${ }^{4,5}$ ) where also the first examples of concrete two-dimensional periodic Fermi surfaces in $\mathbb{R}^{3}$ were considered. The problem of full classification of such trajectories was set by S.P.Novikov in ${ }^{6}$ and considered later in his school (A.V.Zorich, I.A.Dynnikov, S.P.Tsarev).

Let us say here that this problem is rather complicated already for $N=3$ and required non-trivial methods based on topology and dynamical systems theory to be solved completely. The most important breakthroughs in this problem were made in $(\underline{\underline{7}})$ and $(\underline{\underline{11}})$ where very important topological theorems about the non-closed trajectories were proved.

Based on this methods the "topological quantum characteristics" observable in the conductivity of normal metals were introduced in $\frac{15}{}$. These characteristics arise from the geometry of the Fermi surface and have the form of triples of integer numbers connected with the asymptotic behavior of conductivity for $B \rightarrow \infty$ (see also the survey articles 21,26 ). For these physical phenomena an additional property pointed out in ${ }^{15}$ and called later the "Topological resonance" played the important role. We will see here how all these properties can be revealed in the two-dimensional electron gas in a quasiperiodic potential $V(\mathbf{r})$.

Recently the full classification of different trajectories in this situation was finished by I.A.Dynnikov $(\underline{18,22})$ which permits to describe the total picture of the asymptotic behavior of conductivity for $B \rightarrow \infty$ in normal metals with arbitrary complicated dispersion relations $(\underline{26})$.

The case $n=2, N=4$ was started by S.P.Novikov $i^{23}$ where a deep topological theorem analogous to the result of ${ }^{7}$ for this situation was proved. Let us point out here that the case $N=4$ looks very complicated from topological point of view and this theorem is the only deep topological result in this case up to now.

In this paper, however, we work with the coordinate space rather than with the momenta space and consider quasiperiodic functions $V(\mathbf{r})$ where $\mathbf{r}=(x, y)$ plays the role of the ordinary coordinate vector on the plane. In this situation only one plane $\mathbb{R}^{2}$ embedded in $\mathbb{R}^{3}$ or $\mathbb{R}^{4}$ 

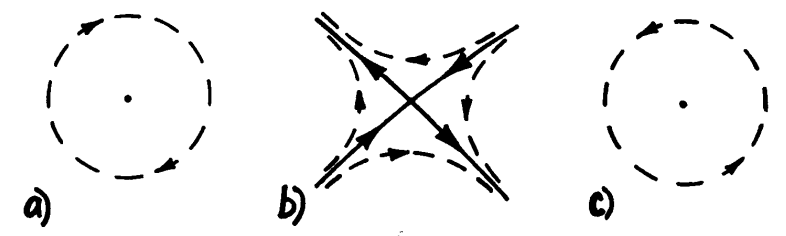

FIG. 1: The level curves of the function $V(\mathbf{r})$ close to the local minimum, the saddle-point and the local maximum of $V(\mathbf{r})$.

will be important. However, also the global characteristics of the total family of potentials corresponding to different parallel planes will arise through the action of the "quasiperiodic group" as we will see below.

\section{THE QUASICLASSICAL TRAJECTORIES AND 2D ELECTRON GAS.}

Let us introduce first the notations for different level curves of potential $V(\mathbf{r})$ according to $18,21,22,26$. We will assume now that the function $V(\mathbf{r})$ is a Morse function on $\mathbb{R}^{2}$, i.e. all the critical points of $V(\mathbf{r})(\nabla V(\mathbf{r})=0)$ are non-degenerate $\left(\operatorname{det}\left\|\partial_{i} \partial_{j} V\right\| \neq 0\right)$. All the critical points of $V(\mathbf{r})$ can then be just the non-degenerate local minima, the non-degenerate saddle points or the non-degenerate local maxima. The local geometry of the level curves close to these critical points are shown at Fig 1, a-c.

Let us call now the level curves of $V(\mathbf{r})$ the quasiclassical drift trajectories according to our further considerations $\underline{56}$ We will also put formally the arrows on the level curves according to the direction of drift in the magnetic field.

Definition 1. We call a trajectory non-singular if it is not adjacent to a critical (saddle) point of the function $V(\mathbf{r})$. The trajectories adjacent to critical points as well as the critical points themselves we call singular trajectories (see Fig. 1).

Definition 2. We call a non-singular trajectory compact if it is closed on the plane. We call a non-singular trajectory open if it is unbounded in $\mathbb{R}^{2}$.

The examples of singular, compact and open nonsingular trajectories are shown on the Fig. 2, a-c.

It is easy to see also that singular trajectories have the measure zero among all the trajectories on the plane.

The geometry of compact trajectories will not be interesting for us here since we are going to consider the

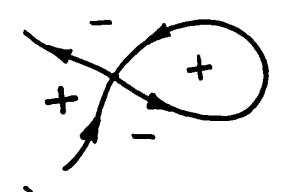

a)

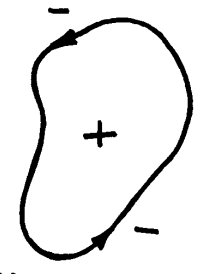

b)

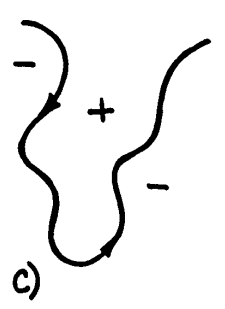

FIG. 2: The singular, compact and open non-singular quasiclassical trajectories. The signs "+ " and "- "show the regions of larger and smaller values of $V(\mathbf{r})$ respectively.

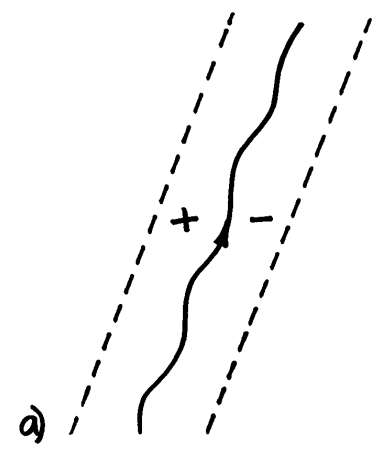

b)

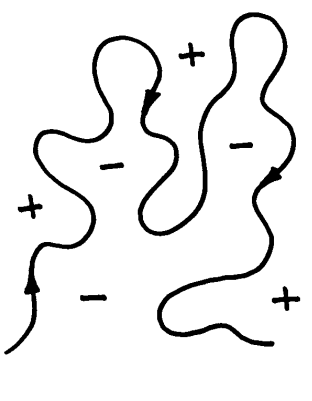

FIG. 3: "Topologically regular" (a) and "chaotic" (b) level curves of the function $V(\mathbf{r})$ in the plane $\mathbb{R}^{2}$.

"geometric" limit corresponding to the long lifetime between two scattering processes. In this limit we assume that every center of drifting cyclotron orbit belongs to the same trajectory for rather long time. This means in particular that all compact trajectories will be passed many times before jumping to another trajectory due to the scattering act. This situation corresponds precisely to the "geometric strong magnetic field limit" considered in $1-3,15,21,26$ where the conductivity in normal metals was studied. However, in our situation this geometric limit does not correspond to strong magnetic field limit as we will see below.

Definition 3. We call an open trajectory topologically regular (corresponding to "topologically integrabl" case) if it lies within a straight line of finite width in $\mathbb{R}^{2}$ and passes through it from $-\infty$ to $\infty$ (see Fig. 3, a). All other open trajectories we will call chaotic (Fig. 3, b).

In the simple case of a periodic function $V(\mathbf{r})(N=2)$ all open trajectories are periodic and we have only "topologically regular" case according to our classification. However, in the quasiperiodic case the situation is much 
more complicated and the chaotic trajectories can exist already for $N=3\left(\frac{12,18}{2}\right)$. These special trajectories can reveal rather complicated stochastic behavior for the general quasiperiodic potentials but fortunately the "generic" open trajectories are still topologically regular for the case $N=3$. Let us point out that this fact was formulated first by S.P.Novikov in form of conjecture and plays now (together with "topological resonance") the crucial role for topological phenomena in normal metals $(15,21,26)$. Here we are also going to consider mainly the "topologically regular" situation for $N=3$ and $N=4$ and we will show that the same "topological numbers" can be observed also for two-dimensional electron gas in specific potentials (quasiperiodic superlattices) built by special experimental techniques in 2-dimensional structures.

Let us describe now the quasiclassical approach for the two-dimensional electron gas which we are going to consider.

The quasiclassical consideration of the $2 \mathrm{D}$ electron gas in the presence of rather strong magnetic field $B$ and a potential $V(\mathbf{r})$ was presented in ${ }^{30}$ in connection with the oscillations of conductivity discovered in 27 . The experiment in ${ }^{27}$ (D.Weiss, K.v.Klitzing, K.Ploog, G.Weimann) used the holographic illumination of high mobility $A l G a A S-G a A S$ heterojunctions at the temperatures $T \leq 4.2 K$. The expanded laser beam was splitten into two parts which gave an interference picture with a period $a$ on a two-dimensional sample. The magnetic field $\mathbf{B}$ was directed normally to the sample and the electron behavior was determined by the magnetic field and the additional periodic potential

$$
V(\mathbf{r})=V(x) \quad, \quad V(x+a)=V(x)
$$

arising after the holographic illumination. The amplitude of $V(x)$ was much smaller than the Fermi energy of the system. Measuring the resistivity in the both directions along and perpendicular to the interference fringes the authors of 27 found the magnetoresistance oscillations in $1 / B$ for magnetic fields smaller than needed for Shubnikov-de-Haas oscillations.

This phenomenon was explained by C.W.J. Beenakker in ${ }^{30}$ from the quasiclassical consideration and called the "Commensurability oscillations". According to the quasiclassical approach the potential $V(x)$ should be averaged over a quasiclassical electron cyclotron orbit with radius $r_{B}=m v_{F} / e B$ on the Fermi level to get the effective averaged potential $\bar{V}(x, B)=V_{B}^{\text {eff }}(x)$ depending on the magnetic field $B$. The condition of weakness of potential $V(x)\left(e V_{r m s} / \epsilon_{F} \ll 1\right.$, where $V_{r m s}$ is the root mean square of $V(x)$ ) should be imposed in this situation. The drift of the center of cyclotron orbit is given then by the equation

$$
\frac{d \mathbf{r}_{0}}{d t}=\frac{e}{B^{2}}\left[\nabla V_{B}^{e f f}\left(\mathbf{r}_{0}\right) \times \mathbf{B}\right]
$$

According to (1) we have a drift of the centers of cyclotron orbits along the level curves of $V_{B}^{e f f}(\mathbf{r})=$ $V_{B}^{e f f}(x)$ with the speed proportional to $\left\|\nabla V_{B}^{e f f}(\mathbf{r})\right\|$ on these curves. As was pointed out in ${ }^{30}$, the drifting motion gives an anisotropic contribution to the conductivity in the plane, depending on the potential $V_{B}^{e f f}(x)$. The crucial role for the magnetoresistance oscillations is played then by a strong dependence of $V_{B}^{e f f}(\mathbf{r})$ on the value of $B$ connected with the commensurability of the cyclotron radius $r_{B}$ (for a given Fermi energy) and the period of potential $a$. The corresponding contribution to the conductivity was thus the oscillating function of $1 / B$ due to the periodic commensurability $2 r_{B}=k a$ with some integer $k$.

The explicit formulae for the conductivity was obtained in ${ }^{30}$ for the model potential having the form $V(x)=V_{x} \cos 2 \pi x / a$. Obviously the main features of this picture will also be true for many generic periodic potentials $V(x)$. Let us also give here the references on the papers $28,29,31,36,38,39,41-46,48,51,54,55$ where different questions connected with this problem were considered (we are sorry for impossibility to give here the complete list of works on this area).

Let us consider now the works where the situation of potentials $V(\mathbf{r})$ modulated both in $x$ and $y$ directions was considered. The potential $V(\mathbf{r})=V(x, y)$ was induced in this case by two independent sets of interference fringes parallel to the $x$ and $y$ axes and the potential $V(x, y)$ was a periodic function in $\mathbb{R}^{2}$ with two periods given by vectors $(a, 0)$ and $(0, a)$.

As was found experimentally $(41,43,44)$ the additional modulation in $y$ direction suppresses the commensurability oscillations in this case. The quasiclassical consideration of this situation was made in 52 where again the drift of electron orbits along the constant energy levels of potential $V(x, y)$ was considered. Two types of the drift trajectories were considered in ${ }^{52}$ :

1) the "pinned orbits" (corresponding to compact energy level curves);

2) the "drifting orbits" (corresponding to unbounded energy level curves in the plane).

As was assumed in ${ }^{52}$ only the contribution of the "drifting orbits" was important for the commensurability oscillations in this case and the "pinned orbits" were unessential for this phenomenon. According to this assumption the suppression of the commensurability oscillations can be explained by the appearance of the "pinned orbits" for the potentials modulated both in $x$ and $y$ directions. Unlike the case of potentials modulated just in $x$ direction, a new condition that the compact trajectories are passed many times by the centers of cyclotron orbits between two scattering acts appeared in 52 . This requirement is similar to the condition of the "geometric strong magnetic field limit" considered in $\underline{\underline{1}}-\underline{\underline{3}}$ for normal metals. However, the limit $B \rightarrow \infty$ does not correspond to the geometric limit in this situation and only $\tau \rightarrow \infty$ should be considered as the geometric limit for this case. Easy to see also that only periodic "drifting orbits" can 
appear for purely periodic potentials $V(x, y)$.

Let us also point out here that the analytic dependence of the resistance on the value of $B$ was also calculated in 52 in the interesting interval for model potentials having few harmonics. This dependence is more complicated compared with the case of $1 \mathrm{D}$ modulated potentials but still reveals the effect of commensurability also in this situation. The mean directions of trajectories appeared in ${ }^{52}$ were parallel to $x$ and $y$ axes and to the diagonal $y=-x$ in different examples. As was also pointed out in ${ }^{52}$ the "drifting orbits" can exist only for potentials with broken rotational symmetry which explains the maximal suppression of the commensurability oscillations for the case of equal modulation intensity in both $x$ and $y$ directions.

In this paper we will not consider in detail the $B$ dependence of conductivity for our more complicated potentials since it should reveal much more complicated behavior in this case. Instead we are going to consider the geometric properties of conductivity tensor in the limit $\tau \rightarrow \infty$ arising from the global geometry of non-singular open trajectories. Namely, we will show that this type of potentials can be considered as a particular case of the quasiperiodic potentials with fixed number of quasiperiods and use the results obtained for the Novikov problem to get the "topological characteristics" of the conductivity in this case. Let us say that this type of "topological quantities" arises in completely different way compared for example with the Hall effect and characterizes the geometry of asymptotic of conductivity tensor (but not its absolute values).

We will not also put any special conditions on potential $V(x, y)$ except the quasiperiodic properties. The formulated results will have a general topological form valid for generic potentials $V(x, y)$.

Before we start the geometric consideration we want to say also that the holographic illumination is not the unique way to produce the superlattice potentials for the two-dimensional electron gas. Let us mention

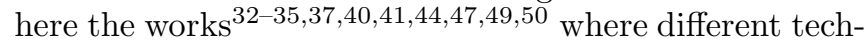
niques using the biasing of the specially made metallic gates and the piezoelectric effect were considered. Both $1 \mathrm{D}$ and $2 \mathrm{D}$ modulated potentials as well as more general periodic potentials with square and hexagonal geometry appeared in this situation. We want to point out that quasiperiodic potentials can be made also by these techniques in the same way using the superposition of several 1D modulations. Actually these techniques give even more possibilities to produce potentials of different types even for the quasiperiodic situation. For example a superposition of a general periodic potential with a generic 1D modulation will give quasiperiodic potentials with 3 quasiperiods which are more general than made just by 3 interference pictures. Also superpositions of two general periodic potentials on the plane will give a class of the quasiperiodic potentials with 4 quasiperiods more general than those which we will consider in detail here. However, we would like for simplicity to restrict ourselves to the simpler pictures of superpositions of $1 \mathrm{D}$
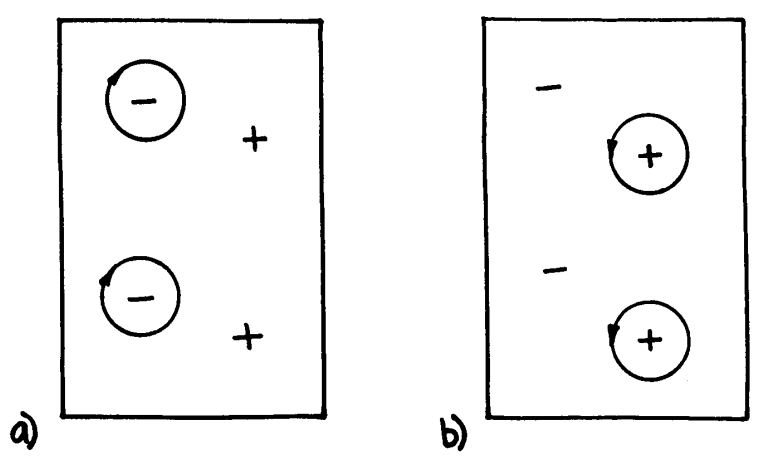

FIG. 4: The level curves of $f(\mathbf{r})$ close to the minimal and maximal values of $f$.

modulation pictures which give already all the features of general behavior. We will also use everywhere the term "interference picture" for the modulation pictures. The general geometrical results will then be true for the other techniques also.

\section{NOVIKOV PROBLEM AND THE GEOMETRIC LIMIT FOR THE CASE OF 3 QUASIPERIODS.}

Let us come now to Novikov problem and start the topological consideration of the level curves of quasiperiodic functions.

We will first describe the situation for an arbitrary periodic potential $V(x, y)$ with some periods $\mathbf{l}_{1}, \mathbf{l}_{2} \in \mathbb{R}^{2}$. This picture is rather simple from the topological point of view but it is convenient to give it here just to introduce the notations and to show the general approach which we are going to use. Let us consider the generic periodic function $f(\mathbf{r})$ on $\mathbb{R}^{2}$ with the values belonging to some interval $\left[f_{\min }, f_{\max }\right]$. We are interested in the form of the level curves $f(\mathbf{r})=c$ where $f_{\min } \leq c \leq f_{\max }$. It is easy to see that for the values of $c$ close to minimal or maximal value of $f$ all such level curves are just small closed loops bounding small regions of lower or higher values of $f$ (see Fig 4, a,b).

It is not difficult to prove also that the extended trajectories (singular or non-singular) always exist in some closed connected "energy interval" $f_{1} \leq c \leq f_{2}\left(f_{\min }<\right.$ $\left.f_{1} \leq f_{2}<f_{\max }\right)$. In generic situation we have $f_{1}<f_{2}$ but for special functions $f(\mathbf{r})$ also the case $f_{1}=f_{2}$ is possible. This fact is actually true for any quasiperiodic function and does not depend on the number of quasiperiods (the proof in $\frac{18}{}$ given for $N=3$ works actually for any $N$ without any change). Every non-singular open trajectory is periodic for a periodic function $f(\mathbf{r})$ with the mean direction given by some integral vector $\mathbf{l}=m_{1} \mathbf{l}_{1}+m_{2} \mathbf{l}_{2}$ of lattice generated by periods $\mathbf{l}_{1}, \mathbf{l}_{2}$. We can see then 


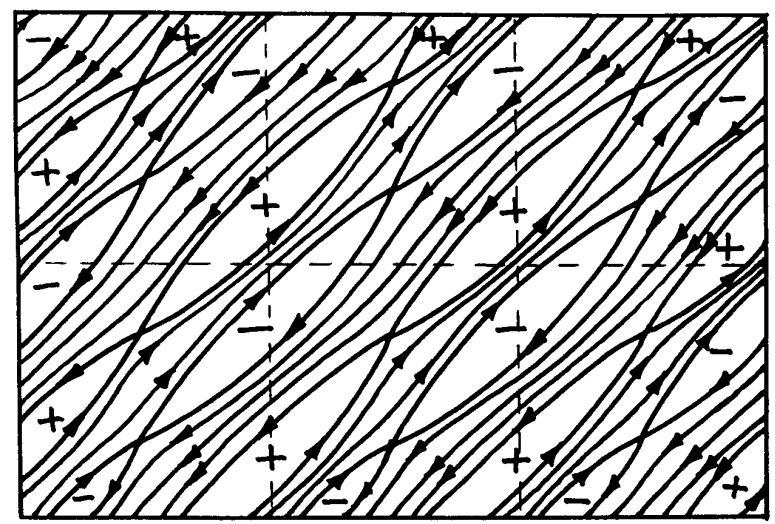

FIG. 5: The layers of open periodic trajectories with the "non-trivial" opposite directions $((1,1)$ and $(-1,-1))$ for the generic periodic function $f(\mathbf{r})$.

that every non-singular open trajectory for periodic $f(\mathbf{r})$ corresponds to "topologically regular" case.

It is easy to see also that there can be only a finite number of energy levels for a periodic Morse function where the singular trajectories can exist. We can claim then that the non-singular open trajectories always exist in the generic case $f_{1}<f_{2}$. The opposite statement is also true since the non-singular open trajectories are stable with respect to a small change of energy level. The typical situation of the generic case with the layers of open trajectories is shown on Fig. 5 .

All the open trajectories do not intersect each other and have a common mean direction passing in both "direct" and the "opposite" way.

The opposite non-generic case $f_{1}=f_{2}$ corresponds to the absence of the non-singular open trajectories in the plane. The typical picture for $f_{1}=f_{2}$ is a "singular net" on the level $f(\mathbf{r})=f_{1}=f_{2}$ and the closed trajectories at all the other levels (Fig. 6). Let us pay here a special attention to the last fact to compare this situation with the more complicated quasiperiodic case.

It follows also that the case $f_{1}=f_{2}$ always takes place for potentials with any kind of rotational symmetry since non-singular open trajectories can not exist in this situation.

Let us give here also the references on the work ${ }^{53}$ where the nice quantization picture based on the topology of periodic quasiclassical drift trajectories in the magnetic field was considered.

The generic periodic potentials $V(\mathbf{r})$ arise in the experiments described above when two independent interference pictures with arbitrary directions of interference fringes are present at the same sample. The potential $V(\mathbf{r})$ is a functional of the total intensity of radiation $I(\mathbf{r})$ and has the same periodicity for any (even nonlocal) translationally invariant dependence of $V(\mathbf{r})$ on the field $I\left(\mathbf{r}^{\prime}\right)$. For simplicity we will put the requirement

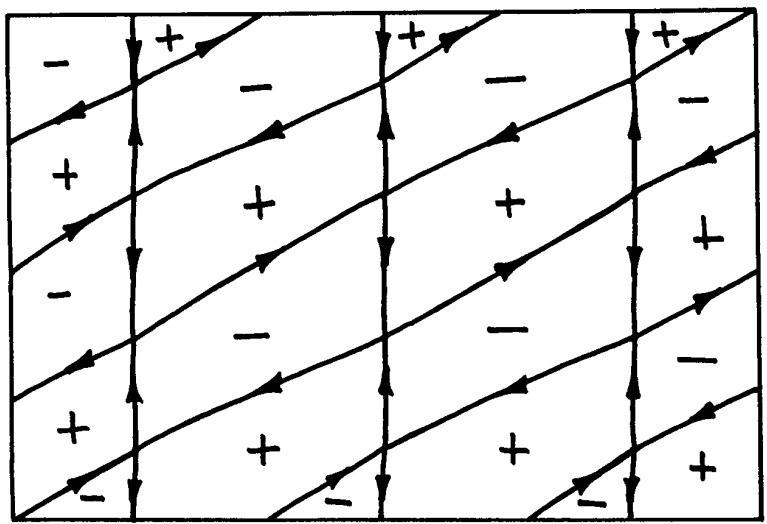

FIG. 6: The singular periodic net on the level $f(\mathbf{r})=f_{1}=f_{2}$ for a non-generic periodic function $f(\mathbf{r})$.

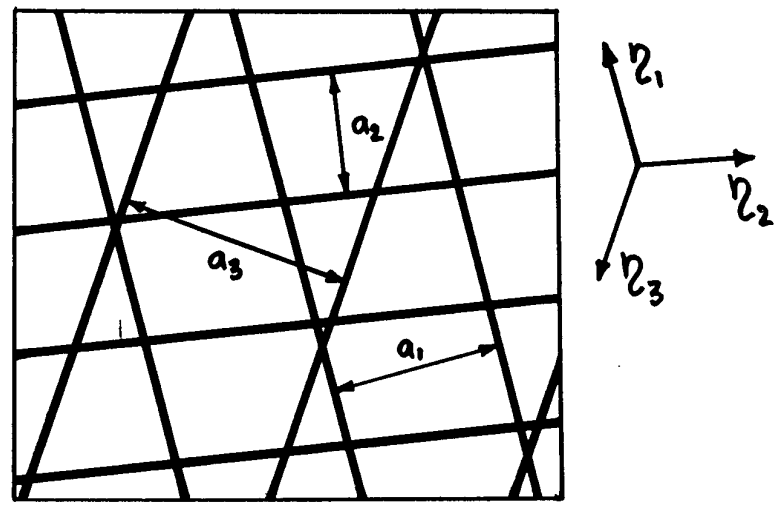

FIG. 7: The schematic sketch of three independent interference pictures on the plane with different periods and intensities.

that the functional $V(\mathbf{r})[I]$ has the variational derivative $\delta V(\mathbf{r}) / \delta I\left(\mathbf{r}^{\prime}\right)$ decreasing for large enough $\left|\mathbf{r}-\mathbf{r}^{\prime}\right|$. We assume also that the functional $V(\mathbf{r})[I]$ is smooth, i.e. gives the smooth function $V(\mathbf{r})$ for any smooth distribution $I\left(\mathbf{r}^{\prime}\right)$.

Let us now come to our main purpose and consider potentials $V(\mathbf{r})$ having more complicated form. Let us have now three independent interference pictures on the plane with three different generic directions of fringes $\boldsymbol{\eta}_{1}, \boldsymbol{\eta}_{2}, \boldsymbol{\eta}_{3}$ and periods $a_{1}, a_{2}, a_{3}$ (see Fig. 7).

The total intensity $I(\mathbf{r})$ will be the sum of intensities

$$
I(\mathbf{r})=I_{1}(\mathbf{r})+I_{2}(\mathbf{r})+I_{3}(\mathbf{r})
$$

of the independent interference pictures.

We assume that there are at least two non-coinciding directions (say $\left.\boldsymbol{\eta}_{1}, \boldsymbol{\eta}_{2}\right)$ among the set $\left(\boldsymbol{\eta}_{1}, \boldsymbol{\eta}_{2}, \boldsymbol{\eta}_{3}\right)$.

Let us draw three straight lines $q_{1}, q_{2}, q_{3}$ with the 


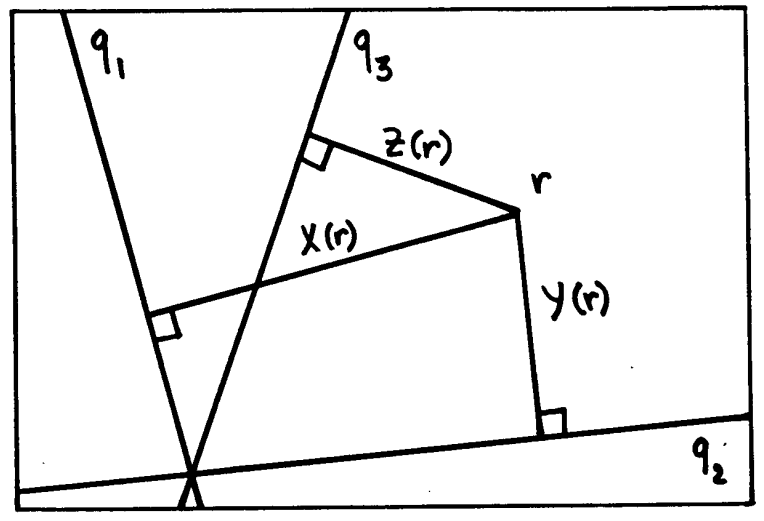

FIG. 8: The coordinates $X(\mathbf{r}), Y(\mathbf{r})$ and $Z(\mathbf{r})$ on the plane.

directions $\boldsymbol{\eta}_{1}, \boldsymbol{\eta}_{2}, \boldsymbol{\eta}_{3}$ and choose the "positive" and "negative" half-planes for every line $q_{i}$ on the plane. Let us consider now three linear functions $X(\mathbf{r}), Y(\mathbf{r}), Z(\mathbf{r})$ on the plane which are the distances from the point $\mathbf{r}$ to the lines $q_{1}, q_{2}, q_{3}$ with the signs " + " or " - " depending on the half-plane for the corresponding line $q_{i}$ (Fig. 8).

The coordinates

$$
\mathbf{R}(\mathbf{r})=(X(\mathbf{r}), Y(\mathbf{r}), Z(\mathbf{r}))
$$

give now a parametric representation of our plane $\Pi^{2}=$ $\mathbb{R}^{2}$ in the 3 -dimensional space $\mathbb{R}^{3}$. The total intensity $I(\mathbf{r})$ can be considered then as the restriction to $\Pi^{2}$ of the periodic function $\hat{I}(X, Y, Z)$ :

$$
\hat{I}(X, Y, Z)=I_{1}(X)+I_{2}(Y)+I_{3}(Z)
$$

corresponding to the lattice in $\mathbb{R}^{3}$ generated by vectors $\left(a_{1}, 0,0\right),\left(0, a_{2}, 0\right),\left(0,0, a_{3}\right)$. The plane $\Pi^{2}$ passes through the origin according to Fig. 8 (although it is not necessary if the lines $q_{1}, q_{2}, q_{3}$ do not intersect at one point in $\mathbb{R}^{2}$ ).

Let us point out here that the standard inner product on the plane $\Pi^{2}$ does not coincide with the product in $\mathbb{R}^{3}$ in this construction (from the metric point of view the plane $\mathbb{R}^{2}$ will be linearly deformed in the embedding $\mathbf{R}=\mathbf{R}(x, y))$. However, the inner product will not be important at all in our further considerations so we don't pay any attention to this fact. Let us just say that it's possible to introduce a special inner product in $\mathbb{R}^{3}$ such that it's restriction on $\Pi^{2}$ will give the standard metric in $\mathbb{R}^{2}$. Nevertheless, all the topological statements will be invariant under the group of all non-degenerate linear transformations and we will not need this construction at all.

We can define now smooth periodic functions $\hat{V}(X, Y, Z)$ and $\hat{V}_{B}^{e f f}(X, Y, Z)$ in $\mathbb{R}^{3}$ such that the functions $V(x, y)$ and $V_{B}^{e f f}(x, y)$ will be the restrictions of
$\hat{V}(X, Y, Z)$ and $\hat{V}_{B}^{e f f}(X, Y, Z)$ on the plane $\Pi^{2}$. Indeed, consider any point $\mathbf{R}=(X, Y, Z) \in \mathbb{R}^{3}$. Let us draw a two-dimensional plane $\Pi^{2 \prime}$ through the point $\mathbf{R}$ parallel to the plane $\Pi^{2}$. We have then the total intensity $I^{\prime}(\mathbf{R})$ in the plane $\Pi^{2 \prime}$ defined as the restriction of $\hat{I}(X, Y, Z)$ on $\Pi^{2 \prime}$. Let us define now the functions $\hat{V}(X, Y, Z)$ and $\hat{V}_{B}^{e f f}(X, Y, Z)$ at the point $\mathbf{R}$ as the corresponding functions defined in the plane $\Pi^{2 \prime}$ passing through $\mathbf{R}$ using the functional $V(\mathbf{r})[I]$ and the averaging over the cyclotron orbits in $\Pi^{2 \prime}$. Easy to see that the functions $\hat{V}(X, Y, Z), \hat{V}_{B}^{e f f}(X, Y, Z)$ are smooth periodic functions in $\mathbb{R}^{3}$ with periods $\left(a_{1}, 0,0\right),\left(0, a_{2}, 0\right),\left(0,0, a_{3}\right)$. Obviously the functions $\left.\hat{V}\right|_{\Pi^{2}}$ and $\left.\hat{V}_{B}^{\text {eff }}\right|_{\Pi^{2}}$ give the required potential $V(\mathbf{r})$ and the effective potential $V_{B}^{e f f}(\mathbf{r})$ in the initial two-dimensional plane $\mathbb{R}^{2}$.

Let us introduce now an important definition of the "quasiperiodic group" acting on the potentials described above. As we saw, our construction gives us an embedding $\Pi^{2}$ of the initial plane $\mathbb{R}^{2}$ in the three-dimensional space $\mathbb{R}^{3}$. At the same time we get the additional planes $\Pi^{2 \prime}$ in $\mathbb{R}^{3}$ parallel to $\Pi^{2}$ with different $I^{\prime}(\mathbf{r}), V^{\prime}(\mathbf{r})$, $V_{B}^{e f f^{\prime}}(\mathbf{r})$ corresponding to the same $\hat{I}(\mathbf{R}), \hat{V}(\mathbf{R})$ and $\hat{V}_{B}^{e f f}(\mathbf{R})$. It is easy to see that the functions $I^{\prime}(\mathbf{r}), V^{\prime}(\mathbf{r})$, $V_{B}^{e f f^{\prime}}(\mathbf{r})$ correspond to the case of three interference pictures with the same mean directions of fringes and periods $\left(\boldsymbol{\eta}_{1}, a_{1}\right),\left(\boldsymbol{\eta}_{2}, a_{2}\right),\left(\boldsymbol{\eta}_{3}, a_{3}\right)$ but with shifted positions of maxima and minima for every interference picture.

Definition 4. We will say that all the potentials $V^{\prime}(\mathbf{r})$ (as well as $V_{B}^{\text {eff'}}(\mathbf{r})$ for every given $B$ ) are related by a "quasiperiodic group" of transformations.

According to the Definition 4 we define the action of a "quasiperiodic group" in $\mathbb{R}^{2}$ as the parallel shifts of the plane $\Pi^{2}$ in the space $\mathbb{R}^{3}$. The "quasiperiodic group" is then a 3-parametric Abelian group isomorphic to 3dimensional torus $\mathbb{T}^{3}=\mathbb{R}^{3} / L$

$$
\begin{gathered}
L=m_{1}\left(a_{1}, 0,0\right)+m_{2}\left(0, a_{2}, 0\right)+m_{3}\left(0,0, a_{3}\right) \\
\left(m_{1}, m_{2}, m_{3}\right) \in \mathbb{Z}^{3}
\end{gathered}
$$

containing the (non-compact) algebraic subgroup of ordinary translations in $\mathbb{R}^{2}$.

As we will see below, this definition will be very convenient in consideration of open trajectories for potentials of this type in $\mathbb{R}^{2}$. Namely, we will see that all the global properties of open trajectories will be the same for all potentials related by the "quasiperiodic group" in the case of generic $\left(\boldsymbol{\eta}_{1}, a_{1}\right),\left(\boldsymbol{\eta}_{2}, a_{2}\right),\left(\boldsymbol{\eta}_{3}, a_{3}\right)$. In other words, for generic $\left(\boldsymbol{\eta}_{1}, a_{1}\right),\left(\boldsymbol{\eta}_{2}, a_{2}\right),\left(\boldsymbol{\eta}_{3}, a_{3}\right)$ the global geometry of open trajectories will not depend on the positions of minima and maxima of the interference pictures and will be defined just by the set $\left(\boldsymbol{\eta}_{1}, a_{1}\right),\left(\boldsymbol{\eta}_{2}, a_{2}\right),\left(\boldsymbol{\eta}_{3}, a_{3}\right)$ and 


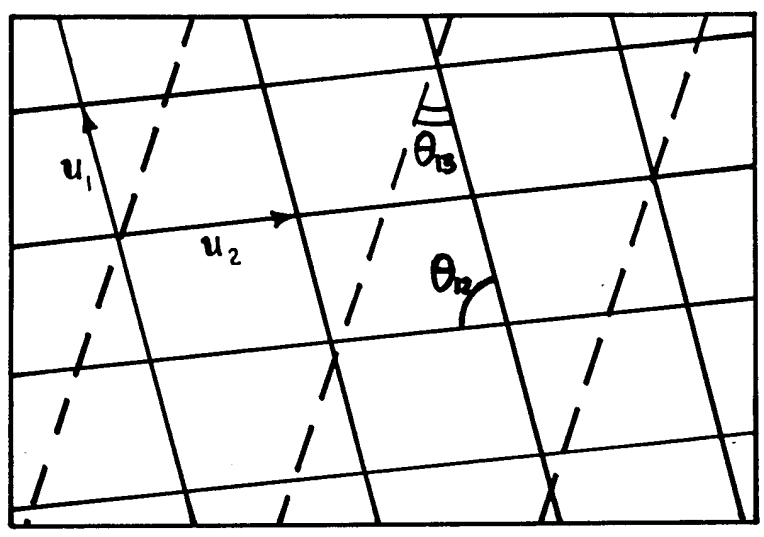

FIG. 9: The periodic picture formed by two sets of parallel interference fringes with common directions $\boldsymbol{\eta}_{1}, \boldsymbol{\eta}_{2}$ and the added third set with direction $\boldsymbol{\eta}_{3}$.

the intensities $I_{1}, I_{2}, I_{3}$ (although the potentials $V(\mathbf{r})$, $V_{B}^{e f f}(\mathbf{r})$ will be different in these cases). Let us say, however, that this property can be broken for special $\left(\boldsymbol{\eta}_{1}, a_{1}\right)$, $\left(\boldsymbol{\eta}_{2}, a_{2}\right),\left(\boldsymbol{\eta}_{3}, a_{3}\right)$ corresponding to purely rational directions of $\Pi^{2}$ in $\mathbb{R}^{3}$.

According to the previous definition we will say that a quasiperiodic potential has irrationality 3 or maximal irrationality if it has no periods in $\mathbb{R}^{2}$. We will say that the potential $V(\mathbf{r})$ has irrationality 2 if it has only one (up to the integer multiplier) period in $\mathbb{R}^{2}$. We will say that the potential $V(\mathbf{r})$ has irrationality 1 if it has two linearly independent periods in $\mathbb{R}^{2}: \frac{57}{5}$ As can be easily seen the last case corresponds to the purely periodic potentials $V(\mathbf{r})$. It is easy to see also that the potentials $V(\mathbf{r})$ of irrationality 3, 2 and 1 correspond to the cases when the plane $\Pi^{2}$ contains no vectors belonging to $L$, just one (up to the integer multiplier) vector belonging to $L$ and two linearly independent vectors belonging to $L$ respectively. Obviously all the potentials related by the "quasiperiodic group" have the same irrationality in the plane.

Let us discuss now briefly the connection of irrationality with the directions and periods of interference pictures in our situation.

We assume as previously that there are at least two different directions of the interference fringes in our picture. The picture given by two corresponding sets of interference fringes is purely periodic in $\mathbb{R}^{2}$ with the periods $\mathbf{u}_{1}$, $\mathbf{u}_{2}$ parallel to $\boldsymbol{\eta}_{1}$ and $\boldsymbol{\eta}_{2}$ respectively (see Fig. 9).

We can see then that the total picture has a period in $\mathbb{R}^{2}$ if some nontrivial integer linear combination

$$
m_{1} \mathbf{u}_{1}+m_{2} \mathbf{u}_{2}, \quad\left(m_{1}, m_{2}\right) \in \mathbb{Z}^{2} /(0,0)
$$

of periods $\mathbf{u}_{1}, \mathbf{u}_{2}$ leaves invariant also the third interference picture corresponding to pair $\left(\boldsymbol{\eta}_{3}, a_{3}\right)$.

The corresponding condition for $m_{1} \mathbf{u}_{1}+m_{2} \mathbf{u}_{2}$ can then be written in the form

$$
\left(m_{1} \mathbf{u}_{1}+m_{2} \mathbf{u}_{2}, \boldsymbol{\xi}_{3}\right)=k a_{3}, \quad k \in \mathbb{Z}
$$

where $\boldsymbol{\xi}_{3}$ is a unit vector orthogonal to $\boldsymbol{\eta}_{3}$ in the plane.

The equation (2) has no nontrivial solutions in generic situation and can be satisfied only for special $\boldsymbol{\eta}_{3}$ and $a_{3}$. It's not difficult to show that for purely rational potentials $V(\mathbf{r})$ (two linearly independent solutions of (2)) the direction $\boldsymbol{\eta}_{3}$ should also correspond to an integer vector in the lattice $L^{\prime}$ generated by vectors $\mathbf{u}_{1}, \mathbf{u}_{2}$ :

$$
L^{\prime}=\left\{m_{1} \mathbf{u}_{1}+m_{2} \mathbf{u}_{2}, \quad\left(m_{1}, m_{2}\right) \in \mathbb{Z}^{2}\right\}
$$

We can put for this case $\boldsymbol{\eta}_{3} \sim m_{1} \mathbf{u}_{1}+m_{2} \mathbf{u}_{2}$ for some integer $m_{1}, m_{2}$. Also the corresponding period $a_{3}$ should satisfy to a special condition in this situation. If we introduce the angles $\theta_{12}, \theta_{13}$ between the directions $\boldsymbol{\eta}_{1}, \boldsymbol{\eta}_{2}$ and $\boldsymbol{\eta}_{1}, \boldsymbol{\eta}_{3}, 0<\theta_{12} \leq \pi / 2,0<\theta_{13} \leq \pi$ (Fig. 9) we can get the relations for $\theta_{13}$ and $a_{3}$ which define all the pairs $\left(\boldsymbol{\eta}_{3}, a_{3}\right)$ corresponding to purely rational potentials $V(\mathbf{r})$ :

$$
\operatorname{tg} \theta_{13}=\frac{m_{2} a_{1} \sin \theta_{12}}{m_{1} a_{2}-m_{2} a_{1} \cos \theta_{12}}
$$

$$
k_{3} a_{3} \sin \theta_{12}=k_{1} a_{1} \sin \left(\theta_{12}+\theta_{13}\right)+k_{2} a_{2} \sin \theta_{13}
$$

where $m_{1}, m_{2}, k_{1}, k_{2}, k_{3} \in \mathbb{Z}, \quad\left(m_{1}, m_{2}\right) \neq(0,0)$, $\left(k_{1}, k_{2}\right) \neq(0,0), k_{3} \neq 0$.

For the case of just one period (irrationality 2 ) we can have either the condition (33) for $\theta_{13}$ ("rational" direction of $\boldsymbol{\eta}_{3}$ ) but with $a_{3}$ not satisfying to (4) or the condition (41) for $a_{3}$ but with $\theta_{13}$ not satisfying to (3).

Easy to see that both cases of irrationality 1 and 2 have the measure zero among all potentials constructed by three arbitrary interference pictures.

As we already said above the case of irrationality 1 corresponds to purely periodic potentials $V(x, y)$. Let us however make here some remark. Namely, for arbitrary periodic potential $V(x, y)$ the corresponding periods $\mathbf{l}_{1}, \mathbf{l}_{2}$ can be much bigger than the values of $a_{1}, a_{2}$, $a_{3}$. We can conclude then that even the "topologically regular" periodic open trajectories can have rather nontrivial structure on rather long distances since the period of trajectory is very big. The width of the straight line containing a periodic trajectory can be also compatible with periods of $V(x, y)$ in this case being quite big with respect to the periods of modulations $a_{1}, a_{2}, a_{3}$. Also the rational mean direction of the periodic trajectories can have "rather big denominator" such that this rationality will not play an essential role in the real picture. Instead, the typical features observable in generic situation of irrationality 3 will appear on the distances smaller than the periods $\left|\mathbf{l}_{1}\right|,\left|\mathbf{l}_{2}\right|$ of potential $V(x, y)$. According to this remark we can actually try to consider potentials of irrationality 1 or 2 as generic potentials of irrationality 
3 if the periods of these potentials are rather big. The special features connected with rationality can then be revealed only for very big values of $\tau$ such that the free motion length is much larger than $\left|\mathbf{l}_{1}\right|,\left|\mathbf{l}_{2}\right|$.

Let us formulate now (in our language) the first theorem about the open trajectories for the quasiperiodic potentials $V(\mathbf{r})$ and $V_{B}^{\text {eff }}(\mathbf{r})$ with 3 quasiperiods corresponding to the first theorem on the Novikov problem proved in ${ }^{7}$ :

Theorem 1. Consider a purely periodic potential $V^{(0)}(\mathbf{r}) \quad\left(\right.$ or $\left.V_{B}^{(0) e f f}(\mathbf{r})\right)$ generated by three independent interference pictures with some parameters $\left(\boldsymbol{\eta}_{1}^{(0)}, a_{1}^{(0)}\right)$, $\left(\boldsymbol{\eta}_{2}^{(0)}, a_{2}^{(0)}\right),\left(\boldsymbol{\eta}_{3}^{(0)}, a_{3}^{(0)}\right)$ satisfying to (3), (4). Then for all the potentials $V(\mathbf{r})$ (and $V_{B}^{\text {eff }}(\mathbf{r})$ ) with parameters $\left(\boldsymbol{\eta}_{1}, a_{1}\right),\left(\boldsymbol{\eta}_{2}, a_{2}\right),\left(\boldsymbol{\eta}_{3}, a_{3}\right)$ close enough to $\left(\boldsymbol{\eta}_{1}^{(0)}, a_{1}^{(0)}\right)$, $\left(\boldsymbol{\eta}_{2}^{(0)}, a_{2}^{(0)}\right),\left(\boldsymbol{\eta}_{3}^{(0)}, a_{3}^{(0)}\right)$ all the open non-singular electron trajectories will correspond to topologically regular case only.

Using the same methods as in ${ }^{7}$ it is possible to prove also that Theorem 1 will be true also for small variations of the intensities $I_{1}(\mathbf{r}), I_{2}(\mathbf{r}), I_{3}(\mathbf{r})$ of the laser beams and the form of the functional $V(\mathbf{r})[I]$.

Let us say here that Theorem 1 makes rather strong statement about generic potentials close to periodic ones. However, the corresponding "stability zones" for parameters $\left(\boldsymbol{\eta}_{1}, \boldsymbol{\eta}_{2}, \boldsymbol{\eta}_{3}, a_{1}, a_{2}, a_{3}\right)$ (and $\left.I_{1}, I_{2}, I_{3}\right)$ depend on the initial values of $\left(\boldsymbol{\eta}_{1}^{(0)}, a_{1}^{(0)}\right),\left(\boldsymbol{\eta}_{2}^{(0)}, a_{2}^{(0)}\right),\left(\boldsymbol{\eta}_{3}^{(0)}, a_{3}^{(0)}\right)$ and become very small for large values of periods $\left|\mathbf{l}_{1}\right|,\left|\mathbf{l}_{2}\right|$ of the initial potential. Due to this reason Theorem 1 can not say anything about arbitrary potential $V(\mathbf{r})$ (with 3 quasiperiods) since we can have the situation when it does not belong to any stability zone corresponding to any rational potential $V^{(0)}(\mathbf{r})$. Nevertheless, this theorem is very important and we will see also that only the result of this type can be formulated for more complicated case of potentials with 4 quasiperiods.

Let us discuss now the general situation of arbitrary potentials $V(\mathbf{r})$ with 3 quasiperiods. We will start first with the generic situation of potentials of irrationality 3 and then discuss additional features which can arise in the cases of irrationality 1 and 2. Let us make here the reference on the survey article $\frac{22}{2}$ where the final theorems in the most complete form were formulated. The development of this problem and the considerations of physical phenomena can be found in ${ }^{\underline{6}-26}$. Let us say also that all the results in ${ }^{22}$ and in all previous papers were formulated in another language using the 3-dimensional topology terminology. We will not discuss here the topological questions in detail and just claim that the following statements can be derived from the topological theorems formulated in 22 .

Theorem 2. Let us fix the value of $B$ and consider the generic quasiperiodic potential $V_{B}^{\text {eff }}(\mathbf{r})$ (of irra- tionality 3) taking the values in some interval $\epsilon_{\min }(B) \leq$ $V_{B}^{e f f}(\mathbf{r}) \leq \epsilon_{\max }(B)$. Then:

1) Open quasiclassical trajectories $V_{B}^{e f f}(\mathbf{r})=c$ always exist either in a connected energy interval

$$
\epsilon_{1}(B) \leq c \leq \epsilon_{2}(B)
$$

$\left(\epsilon_{\min }(B)<\epsilon_{1}(B)<\epsilon_{2}(B)<\epsilon_{\max }(B)\right)$ or just at one energy value $c=\epsilon_{0}(B)$ (i.e. $\epsilon_{1}(B)=\epsilon_{2}(B)=\epsilon_{0}(B)$ ).

2) For the case of a finite interval $\left(\epsilon_{1}(B)<\epsilon_{2}(B)\right)$ all the non-singular open trajectories correspond to topologically regular case, i.e. lie in straight strips of a finite width (Fig. 3, a) and pass through them. All the strips have the same mean directions for all the energy levels $c \in\left[\epsilon_{1}(B), \epsilon_{2}(B)\right]$ such that all the open trajectories are in average parallel to each other for all values of $c$.

3) The values $\epsilon_{1}(B), \epsilon_{2}(B)$ or $\epsilon_{0}(B)$ are the same for all the potentials of irrationality 3 connected by the "quasiperiodic group".

4) For the case of a finite energy interval $\left(\epsilon_{1}(B)<\right.$ $\left.\epsilon_{2}(B)\right)$ all the non-singular open trajectories also have the same mean direction for all the potentials (of irrationality 3) connected by the "quasiperiodic group".

We can see from Theorem 2 that the "topologically integrable" situation is typical also for the case of quasiperiodic functions with 3 quasiperiods being connected with the generic case $\epsilon_{1}(B)<\epsilon_{2}(B)$. Let us say also that for the case of just one energy level $\left(\epsilon_{1}(B)=\epsilon_{2}(B)=\epsilon_{0}(B)\right)$ containing open trajectories both the topologically regular and "chaotic" behavior of open trajectories are possible (see ${ }^{18}$ ). This situation can be compared with the situation of purely periodic potentials where non-singular periodic open trajectories always appear in the case of finite energy interval $\left(\epsilon_{1}(B)>\epsilon_{2}(B)\right)$ but only the periodic "singular nets" are possible for the case $\epsilon_{1}(B)=$ $\epsilon_{2}(B)=\epsilon_{0}(B)$. As we see here quasiperiodic potentials give another possibility in the last case.

Let us consider now the asymptotic behavior of conductivity in the case of topologically regular open trajectories when $\tau \rightarrow \infty$. According to previous papers $(30,45,52)$ we will divide here the conductivity tensor in two parts $\sigma_{0}^{i k}(B)$ and $\Delta \sigma^{i k}(B)$ corresponding to the conductivity without any potential $V(\mathbf{r})$ and an additional contribution due to potential $V(\mathbf{r})$. We have then:

$$
\sigma_{0}^{i k}(B)=\sigma_{0}^{i k}(B)+\Delta \sigma^{i k}(B)
$$

In the approximation of the drifting cyclotron orbits the parts $\sigma_{0}^{i k}(B)$ and $\Delta \sigma^{i k}(B)$ can be interpreted as caused respectively by the (infinitesimally small) difference in the electron distribution function on the same cyclotron orbit (weak angular dependence) and the (infinitesimally small) difference in the occupation of different trajectories by the centers of cyclotron orbits at different points of $\mathbb{R}^{2}$ (on the same energy level) as the linear response to the (infinitesimally) small external field 
E. The asymptotic $\tau \rightarrow \infty$ of both parts $\sigma_{0}^{i k}(B)$ and $\Delta \sigma^{i k}(B)$ can then be written from the same arguments used in $\underline{1}-\underline{3}$ with some additional remarks specific for this situation. We will just say here that the first part $\sigma_{0}^{i k}(B)$ has the standard asymptotic form:

$$
\sigma_{0}^{i k}(B) \sim \frac{n e^{2} \tau}{m^{e f f}}\left(\begin{array}{ll}
\left(\omega_{B} \tau\right)^{-2} & \left(\omega_{B} \tau\right)^{-1} \\
\left(\omega_{B} \tau\right)^{-1} & \left(\omega_{B} \tau\right)^{-2}
\end{array}\right)
$$

for $\omega_{B} \tau \gg 1$ due to the weak angular dependence $(\sim$ $\left.1 / \omega_{B} \tau\right)$ of the distribution function on the same cyclotron orbit. We have then that the corresponding longitudinal conductivity decreases for $\tau \rightarrow \infty$ in all the directions in $\mathbb{R}^{2}$ and the corresponding condition is just $\omega_{B} \tau \gg 1$ in this case.

For the part $\Delta \sigma^{i k}(B)$ the limit $\tau \rightarrow \infty$ should, however, be considered as the condition that every trajectory is passed for rather long time by the drifting cyclotron orbits to reveal its global geometry. Thus another parameter $\tau / \tau_{0}$ where $\tau_{0}$ is the characteristic time of completion of close trajectories should be used in this case and we should put the condition $\tau / \tau_{0} \gg 1$ to have the asymptotic regime for $\Delta \sigma^{i k}(B)$. In this situation the difference between the open and closed trajectories plays the main role and the asymptotic behavior of conductivity can be calculated in the form analogous to that used in ${ }^{1-3}$ for the case of normal metals. Namely:

$$
\Delta \sigma^{i k}(B) \sim \frac{n e^{2} \tau}{m^{e f f}}\left(\begin{array}{cc}
\left(\tau_{0} / \tau\right)^{2} & \tau_{0} / \tau \\
\tau_{0} / \tau & \left(\tau_{0} / \tau\right)^{2}
\end{array}\right)
$$

in the case of closed trajectories and

$$
\Delta \sigma^{i k}(B) \sim \frac{n e^{2} \tau}{m^{e f f}}\left(\begin{array}{cc}
* & \tau_{0} / \tau \\
\tau_{0} / \tau & \left(\tau_{0} / \tau\right)^{2}
\end{array}\right)
$$

$(* \sim 1)$ for the case of open topologically regular trajectories if the $x$-axis coincides with the mean direction of trajectories.

We can see then that only the contribution of open orbits to $\Delta \sigma^{i k}(B)$ remains in (longitudinal) conductivity for $\tau \rightarrow \infty$. Let us say that these formulae give just an asymptotic form of conductivity for $\tau \rightarrow \infty$. In the more precise form they should include also the multipliers proportional to the parts of the phase volume filled by the closed and open trajectories and an appropriate definition of $m^{\text {eff }}$ in this situation. We will not, however, consider this part in detail since we will need only the anisotropy of the tensor $\sigma^{i k}$ in the "geometric limit".

The condition $\tau / \tau_{0} \gg 1$ is much stronger then $\omega_{B} \tau \gg$ 1 in the situation described above just according to the definition of the slow drift of the cyclotron orbits. We can keep then just this condition in our further considerations and assume that the main part of conductivity is given by $\Delta \sigma^{i k}(B)$ in this limit. Easy to see also that the magnetic field $B$ should not be "very strong" in this case.
According to the remarks above we can write now the main part of the conductivity tensor $\sigma^{i k}(B)$ in the limit $\tau \rightarrow \infty$ for the case of topologically regular open orbits. Let us take the $x$ axis along the mean direction of open orbits and take the $y$-axis orthogonal to $x$. The asymptotic form of $\sigma^{i k}, i, k=1,2$ can then be written as:

$$
\sigma^{i k} \sim \frac{n e^{2} \tau}{m^{e f f}}\left(\begin{array}{cc}
* & \tau_{0} / \tau \\
\tau_{0} / \tau & \left(\tau_{0} / \tau\right)^{2}
\end{array}\right), \tau_{0} / \tau \rightarrow 0
$$

where $*$ is some value of order of 1 (constant as $\tau_{0} / \tau \rightarrow$ $0)$.

The asymptotic form of $\sigma^{i k}$ makes possible the experimental observation of the mean direction of topologically regular open trajectories if the value $\tau / \tau_{0}$ is rather big.

Let us introduce here the "topological numbers" characterizing the regular open trajectories introduced first in $^{15}$ for the case of normal metals. We will give a topological definition of these numbers using the action of the "quasiperiodic group" on the quasiperiodic potentials. Let us assume for simplicity that the potential $V_{B}^{e f f}(\mathbf{r})$ is generic and has irrationality 3. We assume that we have the "topologically integrable" situation where the topologically regular open trajectories exist in some finite energy interval $\epsilon_{1}(B) \leq c \leq \epsilon_{2}(B)$. According to Theorem 2 the values $\epsilon_{1}(B), \epsilon_{2}(B)$ and the mean directions of open trajectories are the same for all the potentials constructed from our potential with the aid of the "quasiperiodic group". It follows also from the topological picture that all the topologically regular trajectories are absolutely stable under the action of the "quasiperiodic group" (for the case of irrationality 3) and can just "crawl" in the plane for a continuous action of such transformations.

Let us make now the following transformation:

We take the first interference picture $\left(\left(\boldsymbol{\eta}_{1}, a_{1}\right)\right)$ and shift continuously the interference fringes in the direction of $\operatorname{grad} X(\mathbf{r})$ (orthogonal to $\boldsymbol{\eta}_{1}$ ) to the distance $a_{1}$ keeping two other interference pictures unchanged. Easy to see that we will have at the end the same potentials $V(x, y)$ and $V_{B}^{e f f}(x, y)$ due to the periodicity of the first interference picture with period $a_{1}$. Let us fix now some energy level $c \in\left(\epsilon_{1}(B), \epsilon_{2}(B)\right)$ and look at the evolution of non-singular open trajectories (for $V_{B}^{e f f}(x, y)$ ) while making our transformation. We know that we should have parallel open trajectories in the plane at every time and the initial picture should coincide with the final according to the construction. The form of trajectories can change during the process but their mean direction will be the same according to Theorem $2 . \underline{58}$

We can claim then that every open trajectory will be "shifted" to another open trajectory of the same picture by our continuous transformation. It's not difficult to prove that all the trajectories will then be shifted by the same number of positions $n_{1}$ (positive or negative) which depends on the potential $V_{B}^{\text {eff }}(x, y)$ (Fig. 10).

The number $n_{1}$ is always even since all the trajectories appear by pairs with the opposite drift directions. 


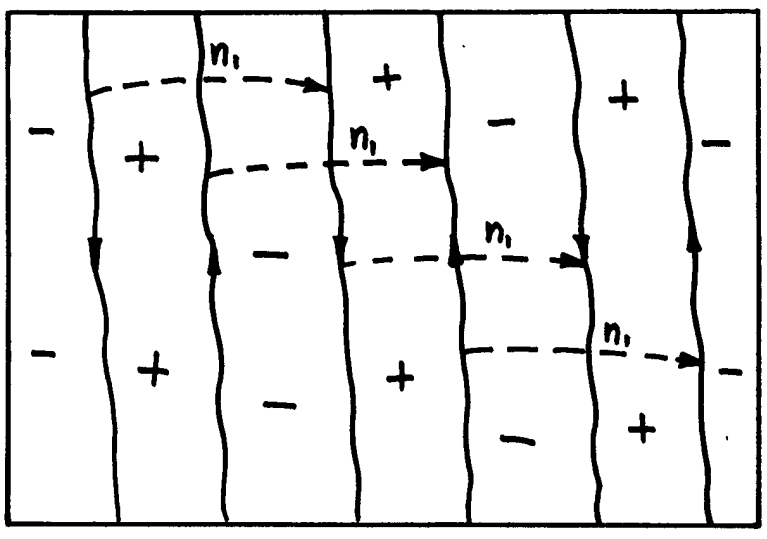

FIG. 10: The shift of "topologically regular" trajectories by a continuous transformation generated by the special path in the "quasiperiodic group".

Let us now do the same with the second and the third sets of the interference fringes and get an integer triple $\left(n_{1}, n_{2}, n_{3}\right)$ which is a topological characteristic of potential $V_{B}^{\text {eff }}(x, y)$ (the "positive" direction of the numeration of trajectories should be the same for all these transformations).

The triple $\left(n_{1}, n_{2}, n_{3}\right)$ (defined up to the common sign) can be represented as:

$$
\left(n_{1}, n_{2}, n_{3}\right)=M\left(m_{1}, m_{2}, m_{3}\right)
$$

where $M \in \mathbb{Z}$ and $\left(m_{1}, m_{2}, m_{3}\right)$ is an indivisible integer triple. Both $M$ and $\left(m_{1}, m_{2}, m_{3}\right)$ have the topological meaning connected with the number of connected components carrying open trajectories in $\mathbb{R}^{3}$ and the homological class of every component in $\mathbb{T}^{3}=\mathbb{R}^{3} / L$ up to the sign.

Let us mention that for periodic potentials $V(x, y)$ made just by two interference pictures with common directions $\boldsymbol{\eta}_{1}, \boldsymbol{\eta}_{2}$ the corresponding transformations are actually equivalent to the shifts along the periods $\mathbf{u}_{2}$ and $\mathbf{u}_{1}$ respectively (Fig. 9). It is not difficult to see that the corresponding numbers $\left(m_{1}, m_{2}\right)$ are equal then (up to the common sign) to $\left(-i_{1}, i_{2}\right)$ where $\left(i_{1}, i_{2}\right)$ is the indivisible integer mean direction of periodic open trajectories in the lattice $L^{\prime}$ generated by vectors $\left\{\mathbf{u}_{1}, \mathbf{u}_{2}\right\}$. Easy to see also that the vectors $\left\{\operatorname{grad} X(\mathbf{r}) / a_{1}, \operatorname{grad} Y(\mathbf{r}) / a_{2}\right\}$ give the dual basis to the basis $\left\{\mathbf{u}_{2}, \mathbf{u}_{1}\right\}$ and the mean direction of open orbits can be defined from the linear equation

$$
m_{1} X(\mathbf{r}) / a_{1}+m_{2} Y(\mathbf{r}) / a_{2}=0
$$

on the plane.

It can be proved that the similar situation also takes place for the topologically regular open trajectories in the case of quasiperiodic potentials $V(\mathbf{r})$. Let us omit here the detailed consideration of the topological picture and just say that the common direction of open trajectories in $\mathbb{R}^{2}$ is defined completely by the triple $\left(m_{1}, m_{2}, m_{3}\right)$. Let us formulate here the corresponding statement:

Theorem 3. Consider the functions

$X^{\prime}(\mathbf{r})=X(\mathbf{r}) / a_{1}, Y^{\prime}(\mathbf{r})=Y(\mathbf{r}) / a_{2}, \quad Z^{\prime}(\mathbf{r})=Z(\mathbf{r}) / a_{3}$

in $\mathbb{R}^{2}$. The mean direction of the regular open trajectories is given by the linear equation:

$$
m_{1} X^{\prime}(x, y)+m_{2} Y^{\prime}(x, y)+m_{3} Z^{\prime}(x, y)=0
$$

where $\left(m_{1}, m_{2}, m_{3}\right)$ is the indivisible integer triple introduced above.

The triples $\left(m_{1}, m_{2}, m_{3}\right)$ coincide precisely with the "Topological Quantum Numbers" introduced in ${ }^{15}$ for the conductivity in normal metals. Let us say that the condition (6) determines completely the numbers $\left(m_{1}, m_{2}, m_{3}\right)$ (from the mean direction of open trajectories) for potentials of irrationality 3 . This fact permits to extract the values of $\left(m_{1}, m_{2}, m_{3}\right)$ from the direct conductivity observations using the anisotropy of tensor $\sigma^{i k}(B)$. (The formula (6) is also true for the case of so-called "stable" open trajectories for potentials of irrationality 1 and 2 (see below). The triple $\left(m_{1}, m_{2}, m_{3}\right)$ generally speaking may not be defined uniquely from the mean directions of open trajectories in these cases and the arguments based on quasiperiodic group play then the main role in the definition of $\left(m_{1}, m_{2}, m_{3}\right)$. However, it can be measured from the direct conductivity observations also in these cases due to the stability of these numbers with respect to the small change of parameters $\left(\boldsymbol{\eta}_{1}, a_{1}\right),\left(\boldsymbol{\eta}_{2}, a_{2}\right)$, $\left(\boldsymbol{\eta}_{3}, a_{3}\right)$.)

A very important property of the integer triples $\left(m_{1}, m_{2}, m_{3}\right)$ is their stability with respect to the small variations of all the parameters $\boldsymbol{\eta}_{1}, \boldsymbol{\eta}_{2}, \boldsymbol{\eta}_{3}, a_{1}$, $a_{2}, a_{3}, I_{1}, I_{2}, I_{3}$ and even of the form of dependence $V(\mathbf{r})[I]$. This means that the space of parameters $\left(\boldsymbol{\eta}_{1}, \boldsymbol{\eta}_{2}, \boldsymbol{\eta}_{3}, a_{1}, a_{2}, a_{3}, I_{1}, I_{2}, I_{3}\right)$ where the situation $\epsilon_{2}(B)>\epsilon_{1}(B)$ for the energy interval containing the open trajectories takes place can be divided into different "stability zones" $\Gamma_{\alpha}$ where the relations (6) are valid for generic $V_{B}^{e f f}(\mathbf{r})$ with the same values of $\left(m_{1}^{\alpha}, m_{2}^{\alpha}, m_{3}^{\alpha}\right)$. Let us emphasize here that the mean directions of open trajectories are different for different values of parameters even within the same stability zone $\Gamma_{\alpha}$ and the equation (6) gives a fixed relation of these directions with the directions and periods of the interference fringes for a given stability zone.

The zones $\Gamma_{\alpha}$ form an everywhere dense set in the total space of parameters and in general we can have an infinite number of zones parameterized by the numbers $\left(m_{1}^{\alpha}, m_{2}^{\alpha}, m_{3}^{\alpha}\right)$. The triples $\left(m_{1}^{\alpha}, m_{2}^{\alpha}, m_{3}^{\alpha}\right)$ form some subset of all possible integer triples $\left(m_{1}, m_{2}, m_{3}\right)$ (defined up 
to the common sign) and give an important topological characteristic of the potentials $V_{B}^{\text {eff }}(\mathbf{r})$ made by 3 interference pictures. The sizes of zones $\Gamma_{\alpha}$ decrease for the big numbers $\left(m_{1}^{\alpha}, m_{2}^{\alpha}, m_{3}^{\alpha}\right)$ and the total set $\left\{\cup \Gamma_{\alpha}\right\}$ gives a rather complicated subset in the space of parameters $\left(\boldsymbol{\eta}_{1}, \boldsymbol{\eta}_{2}, \boldsymbol{\eta}_{3}, a_{1}, a_{2}, a_{3}, I_{1}, I_{2}, I_{3}\right)$. Let us say also that the topologically regular open trajectories are also stable with respect to any variation of potential $V_{B}^{\text {eff }}(\mathbf{r})$ small enough which makes possible to observe them also for slightly imperfect quasiperiodic potentials $V(\mathbf{r})$.

Before starting with special possibilities for the periodic (irrationality 1) or "partly periodic" (irrationality 2) potentials we will say here some words about the "chaotic" behavior of open trajectories possible in the case $\epsilon_{1}(B)=\epsilon_{2}(B)=\epsilon_{0}(B)$. Let us say that for $\epsilon_{1}(B)=\epsilon_{2}(B)$ both the situations of topologically regular and chaotic behavior of open trajectories are possible in the quasiperiodic case. The first situation always takes place when the corresponding set $\left(\boldsymbol{\eta}_{1}, \boldsymbol{\eta}_{2}, \boldsymbol{\eta}_{3}, a_{1}, a_{2}, a_{3}, I_{1}, I_{2}, I_{3}\right)$ belongs to a boundary of some stability zone $\Gamma_{\alpha}$ in the space of parameters. In this case all the non-singular open trajectories are topologically regular and correspond to the same numbers $\left(m_{1}^{\alpha}, m_{2}^{\alpha}, m_{3}^{\alpha}\right)$. Another situation arises when the set $\left(\boldsymbol{\eta}_{1}, \boldsymbol{\eta}_{2}, \boldsymbol{\eta}_{3}, a_{1}, a_{2}, a_{3}, I_{1}, I_{2}, I_{3}\right)$ is an accumulation point for the zones $\Gamma_{\alpha}$ but does not belong to the boundary of any $\Gamma_{\alpha}$. In this situation much more complicated chaotic behavior of open orbits appear at the energy level $V_{B}^{e f f}(\mathbf{r})=\epsilon_{0}(B)$. Obviously the "chaotic" behavior can be possible only for potentials of irrationality 2 or 3 . Let us say also that the cases of irrationality 2 (Tsarev chaotic behavior) and 3 (Dynnikov chaotic behavior) demonstrate completely different types of chaotic behavior in this situation.

The first example of chaotic open trajectory was constructed by S.P.Tsarev $(\underline{12,18})$ for the case of irrationality 2. The corresponding chaotic trajectory, however, has an asymptotic direction but can not be bounded by any straight strip of the finite width in $\mathbb{R}^{2}$. As was later proved by I.A.Dynnikov $(\underline{18})$ this situation always takes place for chaotic trajectories in the case of irrationality 2 . The asymptotic behavior of conductivity tensor reveals also the strong anisotropy for large $\tau$ in this situation with slightly different from (5) dependence on $\tau$ for $\tau \rightarrow \infty$.

More complicated chaotic trajectories were constructed by I.A.Dynnikov (18) for the case of irrationality 3 (the approximate form of such kind of trajectories is shown on Fig. 3, b). The trajectories of this second kind don't have any asymptotic direction in $\mathbb{R}^{2}$ "walking everywhere" in the plane. The form of conductivity tensor for this type of trajectories was suggested in 20 and is more complicated then (5). We will not discuss here all the details and just say that the conductivity decreases in this case in all directions for $\tau \rightarrow \infty$ as some non-integer powers of $\tau \stackrel{59}{\underline{5}}$ Let us also add here that all the chaotic trajectories are completely unstable with respect to small variations of parameters $\left(\boldsymbol{\eta}_{1}, \boldsymbol{\eta}_{2}, \boldsymbol{\eta}_{3}, a_{1}, a_{2}, a_{3}, I_{1}, I_{2}, I_{3}\right)$ (but remain chaotic with the "same geometric properties" under the action of the "quasiperiodic group").

Let us discuss also the $B$-dependence of tensor $\sigma^{i k}(B)$ for the limit $\tau \rightarrow \infty$. The value of $B$ belongs here to some interval $B_{1} \leq B \leq B_{2}$ such that both the drifting orbits approximation and the condition $\tau / \tau_{0} \gg 1$ (as well the absence of quantum oscillations) are true. The effective potential $V_{B}^{\text {eff }}(\mathbf{r})$ is a function of $B$ in this case and the geometry of trajectories depends on $B$ through the potential $V_{B}^{e f f}(\mathbf{r})$. Let us just say here that it can be also proved using topological considerations that the topologically regular open orbits are also "locally stable" with respect to small variations of $B$. However, for rather big changes of value of $B$ it's possible to have "jumps" in this picture and get different mean directions of open trajectories (as well as chaotic cases) in different parts of the interval $\left[B_{1}, B_{2}\right]$. Let us add also that the structure of $B$-dependence can be rather complicated in this case containing an infinite number of small subintervals with very big numbers $\left(m_{1}, m_{2}, m_{3}\right)$ as well as the chaotic cases.

Actually, all the theorems 1-3 can be reformulated in the same form if we add the parameter $B$ to parameters $\boldsymbol{\eta}_{1}, \boldsymbol{\eta}_{2}, \boldsymbol{\eta}_{3}, a_{1}, a_{2}, a_{3}, I_{1}, I_{2}, I_{3}$ introduced above. The probability of "jumps" will then increase for small stability zones $\Gamma_{\alpha}$ corresponding to big numbers $\left(m_{1}, m_{2}, m_{3}\right)$ and the $B$-dependence of $\sigma^{i k}(B)$ will depend strongly on the part of the phase space. The chaotic trajectories will be completely unstable with respect to any small variations of $B$.

In the same way we can consider the "stability zones" $\Gamma_{\alpha}^{e x t}$ in the extended space of parameters including the value of the magnetic field $B$. The total set $\left\{\cup \Gamma_{\alpha}^{e x t}\right\}$ will have then an analogous structure containing in general an infinite number of zones $\Gamma_{\alpha}^{e x t}$ and triples $\left(m_{1}, m_{2}, m_{3}\right)$ being everywhere dense in the total set of parameters.

Let us formulate now the general conjecture of S.P.Novikov about the chaotic cases for potentials with 3 quasiperiods. In our situation we will assume that potentials are parameterized by parameters

$$
\left(\boldsymbol{\eta}_{1}, \boldsymbol{\eta}_{2}, \boldsymbol{\eta}_{3}, a_{1}, a_{2}, a_{3}, I_{1}, I_{2}, I_{3}\right)
$$

or

$$
\left(\boldsymbol{\eta}_{1}, \boldsymbol{\eta}_{2}, \boldsymbol{\eta}_{3}, a_{1}, a_{2}, a_{3}, I_{1}, I_{2}, I_{3}, B\right)
$$

and maybe some additional parameters characterizing the functional $V(\mathbf{r})[I]$.

Novikov conjecture. The set of parameters corresponding to the chaotic behavior of open orbits has measure zero in the total space of parameters.

Let us point out that Novikov conjecture was strictly proved in the important case when only the quasiclassical 


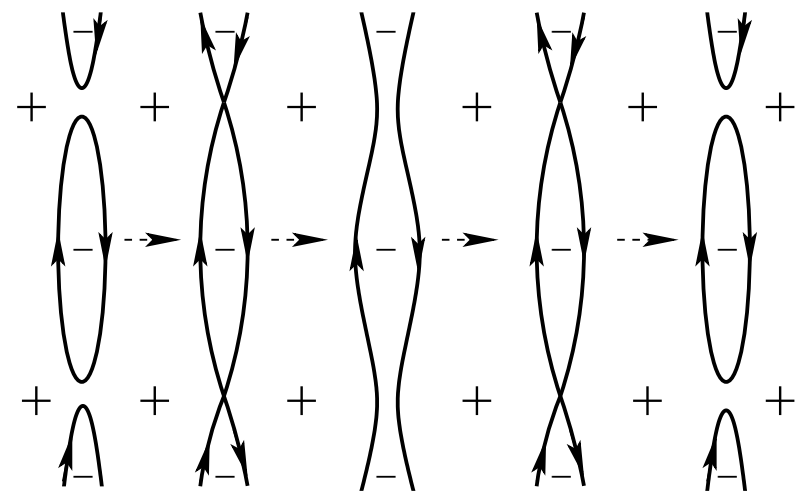

FIG. 11: The arising and the disappearance of periodic trajectories under the action of the "quasiperiodic group" for potentials of irrationality 2 or 1 .

trajectories belonging to some fixed energy level are taken into account $\left(\frac{18}{)}\right)$. This is precisely the situation arising in the conductivity in normal metals where only the trajectories close to the Fermi surface are important. The more general situation was also investigated numerically $\left(\frac{25}{}\right)$ for the case of the special analytic dispersion relations where the Novikov conjecture was also confirmed. However, the general proof of Novikov conjecture for arbitrary set of parameters is still unknown.

We will point out now some additional possibilities which can arise in the non-generic case of potentials of irrationality 1 or 2 ( $\mathrm{see}^{22}$ for detailed mathematical considerations).

Let us start with the case of irrationality 2 when only one period $\mathbf{l}$ (up to an integer multiplier) exists in $\mathbb{R}^{2}$. All the parts (1)-(4) of Theorem 2 are also true for potentials of irrationality 2. We need, however, to make one remark about the situation when the mean direction of the "topologically regular" open trajectories coincides with the period $\mathbf{l}$ of potential. Easy to see that the open trajectories are actually periodic in $\mathbb{R}^{2}$ in this case with the same period 1 . In this situation some "additional pairs" of periodic open trajectories can arise and disappear under the action of the "quasiperiodic group". These pairs arise from periodic sets of closed trajectories under the changing of positions of interference fringes (with the same $\left(\boldsymbol{\eta}_{1}, \boldsymbol{\eta}_{2}, \boldsymbol{\eta}_{3}, a_{1}, a_{2}, a_{3}\right)$ ) and disappear in the same way (Fig. 11).

The trajectories of this kind are unstable with respect to small variations of parameters $\left(\boldsymbol{\eta}_{1}, \boldsymbol{\eta}_{2}, \boldsymbol{\eta}_{3}, a_{1}, a_{2}, a_{3}\right)$ and will be destroyed after any small variation which does not conserve the period $\mathbf{l}$ of potential. These trajectories always present for all the potentials of irrationality 2 connected by the "quasiperiodic group" (on the same energy levels) if they exist at least for one of them. However, these trajectories can "jump" over the two-dimensional plane $\mathbb{R}^{2}$ disappearing in one place and arising in another under the action of group transformations. We can call these trajectories "partly stable" (or also "jumping") in contrary to the absolutely stable ("crawling") trajecto- ries described above. It can be proved also that the phase volume corresponding to both stable and "jumping" open trajectories is also the same for potentials connected by the quasiperiodic group in this situation.

The triple of the integer numbers $\left(n_{1}, n_{2}, n_{3}\right)$ can be defined here in the same way as in the case of irrationality 3 but these additional pairs of trajectories should be completely ignored when the action of the "quasiperiodic group" is considered. The motion of stable open orbits (which always exist in this situation) gives then the same topological numbers $M$ and $\left(m_{1}, m_{2}, m_{3}\right)$ as for close generic potentials.

All the trajectories still have the same mean direction in this situation and the asymptotic form (5) for $\tau \rightarrow \infty$ is also true in this case. The formula (6) is also valid for the directions of open trajectories with the same $\left(m_{1}, m_{2}, m_{3}\right)$. At the end we mention that the situation described above can arise only if the mean directions of stable open orbits coincide with the period $\mathbf{l}$ of potential $V_{B}^{e f f}(\mathbf{r})$ and is absent if it is not so. As we also mentioned already the chaotic behavior is also possible for potentials of irrationality 2 but it is always simpler than for the irrationality 3 potentials.

Let us now say some words about the purely periodic potentials (irrationality 1) which can also appear for special $\left(\boldsymbol{\eta}_{1}, \boldsymbol{\eta}_{2}, \boldsymbol{\eta}_{3}, a_{1}, a_{2}, a_{3}\right)$. As we already said all the open trajectories are purely periodic in this case and only "topologically integrable" situation is possible. We also mentioned already that the extended trajectories can exist here either in the continuous energy interval $\epsilon_{1}(B) \leq c \leq \epsilon_{2}(B)$ or just at one energy level $c=\epsilon_{0}(B)$ (periodic singular nets). All the values $\epsilon_{1}(B)$, $\epsilon_{2}(B), \epsilon_{0}(B)$, however, are not necessarily invariant here with respect to the "quasiperiodic group" action and can be different for different potentials connected by the "quasiperiodic group" transformations. Also the mean directions of open orbits can be different for two potentials belonging to the same orbit of the "quasiperiodic group".

We have then that unlike the cases of irrationality 3 or 2 the positions of interference minima and maxima can be important here for the conductivity behavior and the parameters $\left(\boldsymbol{\eta}_{1}, a_{1}\right),\left(\boldsymbol{\eta}_{2}, a_{2}\right),\left(\boldsymbol{\eta}_{3}, a_{3}\right)$ do not determine the picture completely. It can be proved, however, that a change of the mean directions of open orbits is possible only if the case of "periodic singular net" takes place at least for one (actually at least for two) of potentials belonging to the same orbit of the "quasiperiodic group". We can assume then that this situation takes place only if the periodic potential is prepared specially to have this property and it does not take place for potentials with rather big periods $\mathbf{l}_{1}, \mathbf{l}_{2}$ appeared "by chance" in the modulation picture. Thus we can assume that periodic potentials with rather big $\mathbf{l}_{1}, \mathbf{l}_{2}$ arising "by chance" can be considered actually as generic potentials on the physical level of strictness and do not give any special features. 


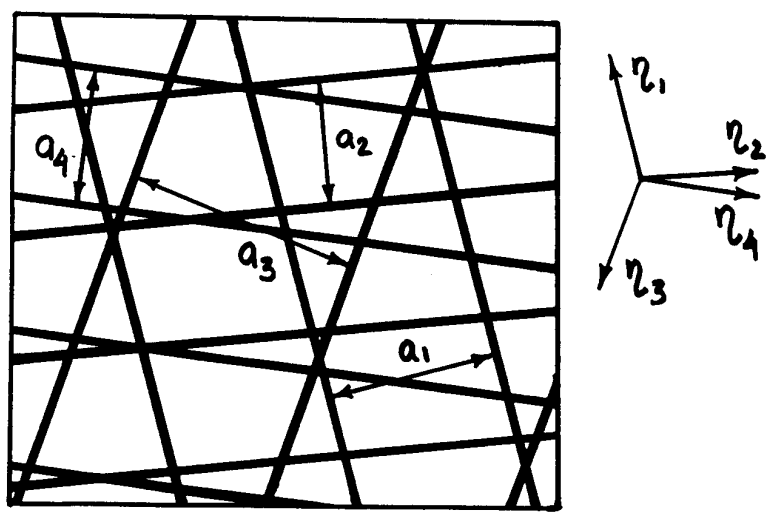

FIG. 12: A potential with 4 quasiperiods made by 4 independent sets of interference fringes with directions $\boldsymbol{\eta}_{1}, \boldsymbol{\eta}_{2}, \boldsymbol{\eta}_{3}, \boldsymbol{\eta}_{4}$ and periods $a_{1}, a_{2}, a_{3}, a_{4}$.

\section{NOVIKOV PROBLEM FOR THE CASE OF POTENTIALS WITH 4 QUASIPERIODS.}

Let us consider now more complicated case when $N=$ 4 and we have a potential made by 4 independent interference pictures (Fig. 12).

The situation in this case is more complicated than in the case $N=3$ and no general classification of open trajectories exists at the time. We will present here the theorem of S.P. Novikov ${ }^{23}$ which gives a statement analogous to Zorich theorem (Theorem 1) in this situation.

Like in the previous case we define here an embedding of the plane $\mathbb{R}^{2}$ in the four-dimensional space $\mathbb{R}^{4}$ using the functions $X(\mathbf{r}), Y(\mathbf{r}), Z(\mathbf{r}), W(\mathbf{r})$ defined in the same way for four interference pictures. We will need also the functions $X^{\prime}(\mathbf{r}), Y^{\prime}(\mathbf{r}), Z^{\prime}(\mathbf{r}), W^{\prime}(\mathbf{r})$ defined as

$$
\begin{aligned}
& X^{\prime}(\mathbf{r})=X(\mathbf{r}) / a_{1}, Y^{\prime}(\mathbf{r})=Y(\mathbf{r}) / a_{2} \\
& Z^{\prime}(\mathbf{r})=Z(\mathbf{r}) / a_{3}, W^{\prime}(\mathbf{r})=W(\mathbf{r}) / a_{4}
\end{aligned}
$$

(in the same way as previously for the case $N=3$ ).

The "total intensity function" $\hat{I}(\mathbf{R}), \mathbf{R} \in \mathbb{R}^{4}$ is defined here as

$$
\hat{I}(\mathbf{R})=I_{1}(X)+I_{2}(Y)+I_{3}(Z)+I_{4}(W)
$$

and is a periodic function with periods $\left(a_{1}, 0,0,0\right)$, $\left(0, a_{2}, 0,0\right),\left(0,0, a_{3}, 0\right),\left(0,0,0, a_{4}\right)$ in $\mathbb{R}^{4}$. The "big potentials" $\hat{V}(\mathbf{R})$ and $\hat{V}_{B}^{\text {eff }}(\mathbf{R})$ are also defined for every point $\mathbf{R} \in \mathbb{R}^{4}$ through the functional $V(\mathbf{R})[I]$ and the averaging over the cyclotron orbits in the plane $\Pi^{2 \prime} \in \mathbb{R}^{4}$ passing through the point $\mathbf{R}$ and parallel to the initial plane $\Pi^{2}$. Easy to see again that the functions $\hat{V}(\mathbf{R})$, $\hat{V}_{B}^{e f f}(\mathbf{R})$ are smooth 4 -periodic functions in $\mathbb{R}^{4}$ and the potentials $V(\mathbf{r}), V_{B}^{e f f}(\mathbf{r})$ are the restrictions of $\hat{V}(\mathbf{R})$ and $\hat{V}_{B}^{e f f}(\mathbf{R})$ on the plane $\Pi^{2}$ embedded in $\mathbb{R}^{4}$. We can define again the action of the "quasiperiodic group" on the potentials $V(\mathbf{r}), V_{B}^{e f f}(\mathbf{r})$ which is now isomorphic to the four-dimensional torus $\mathbb{T}^{4}=\mathbb{R}^{4} / L$ where $L$ is an integer lattice generated by vectors $\left(a_{1}, 0,0,0\right),\left(0, a_{2}, 0,0\right)$, $\left(0,0, a_{3}, 0\right),\left(0,0,0, a_{4}\right)$. Let us mention also that the action of this group can be defined here in the same way as the shifts of positions of minima and maxima of the interference fringes keeping the same the directions $\boldsymbol{\eta}_{1}$, $\boldsymbol{\eta}_{2}, \boldsymbol{\eta}_{3}, \boldsymbol{\eta}_{4}$ and periods $a_{1}, a_{2}, a_{3}, a_{4}$.

Again the statement that the open trajectories always exist either on the connected energy interval $\epsilon_{1}(B) \leq c \leq$ $\epsilon_{2}(B)$ or just at one energy level $\epsilon_{0}(B)$ for any $V_{B}^{e f f}(\mathbf{r})$ is true for the case of 4 quasiperiods. It can be also proved that the values of $\epsilon_{1}(B), \epsilon_{2}(B)$ or $\epsilon_{0}(B)$ are the same for generic potentials belonging to the same orbit of the "quasiperiodic group". Moreover, the global behavior of open trajectories is also the same in this case for all such potentials and the asymptotic behavior of conductivity (which is apriori unknown here for the general case) does not depend on the positions of maxima and minima for the fixed generic $\left(\boldsymbol{\eta}_{1}, a_{1}\right),\left(\boldsymbol{\eta}_{2}, a_{2}\right),\left(\boldsymbol{\eta}_{3}, a_{3}\right)$, $\left(\boldsymbol{\eta}_{4}, a_{4}\right)$. This properties, however, can be destroyed for the specially made periodic potentials $V(\mathbf{r})$ like in the case of 3 quasiperiods.

Let us consider now a purely periodic potential $V(\mathbf{r})$ formed now by four interference pictures. We assume again that at least two (say $\boldsymbol{\eta}_{1}, \boldsymbol{\eta}_{2}$ ) directions of interference fringes are not parallel to each other and give a double-periodic picture in the plane like in the case of potentials with 3 quasiperiods. Let us introduce the angles $\left(\theta_{12}, \theta_{13}, \theta_{14}\right)$ between the directions $\boldsymbol{\eta}_{1}$ and $\boldsymbol{\eta}_{2}, \boldsymbol{\eta}_{3}$, $\boldsymbol{\eta}_{4}$ in the same way as in the case of three interference pictures. From the requirement of periodicity we then will have the same requirements (3)-(4) for the angles $\theta_{13}, \theta_{14}$ and the periods $a_{3}, a_{4}$ with some integer numbers $m_{1}^{\prime}, m_{2}^{\prime}, k_{1}^{\prime}, k_{2}^{\prime}, k_{3}^{\prime}$ (for $\theta_{13}$ and $a_{3}$ ) and $m_{1}^{\prime \prime}, m_{2}^{\prime \prime}, k_{1}^{\prime \prime}$, $k_{2}^{\prime \prime}, k_{3}^{\prime \prime}$ (for $\theta_{14}$ and $a_{4}$ ). Easy to prove that these conditions are also sufficient for the periodicity of the resulting potential $V(\mathbf{r})$.

Theorem of Novikov permits to formulate here the following property of the potentials $V_{B}^{e f f}(\mathbf{r})$ close enough to purely periodic potentials:

Theorem 4. Consider a purely periodic potential $V_{B}^{(0) e f f}(\mathbf{r})$ built by four interference pictures with the directions and periods $\left(\boldsymbol{\eta}_{1}^{(0)}, a_{1}^{(0)}\right),\left(\boldsymbol{\eta}_{2}^{(0)}, a_{2}^{(0)}\right),\left(\boldsymbol{\eta}_{3}^{(0)}, a_{3}^{(0)}\right)$, $\left(\boldsymbol{\eta}_{4}^{(0)}, a_{4}^{(0)}\right)$. Then there exists such small region $\Gamma$ of parameters $\boldsymbol{\eta}_{1}, \boldsymbol{\eta}_{2}, \boldsymbol{\eta}_{3}, \boldsymbol{\eta}_{4}, a_{1}, a_{2}, a_{3}, a_{4}, I_{1}, I_{2}, I_{3}, I_{4}$ containing the initial potential $V_{B}^{(0) e f f}(\mathbf{r})$ that for all the generic potentials $V_{B}^{\text {eff }}(\mathbf{r})$ corresponding to the point of $\Gamma$ the following statements are true:

1) All the non-singular open trajectories lie in the straight strips of finite width and pass through them.

2) All the regular trajectories have the mean direction 
in $\mathbb{R}^{2}$ given by the equation

$$
m_{1} X^{\prime}(\mathbf{r})+m_{2} Y^{\prime}(\mathbf{r})+m_{3} Z^{\prime}(\mathbf{r})+m_{4} W^{\prime}(\mathbf{r})=0
$$

with some integer (indivisible) 4-tuple $\left(m_{1}, m_{2}, m_{3}, m_{4}\right)$ which is the same for all the (generic) points of "stability zone" $\Gamma$.

3) The mean direction of open trajectories are the same for generic potentials belonging to the same orbit of "quasiperiodic group".

Using Novikov theorem it's possible to prove also that the 4-tuples $\left(m_{1}, m_{2}, m_{3}, m_{4}\right)$ can be also defined through the action of "quasiperiodic group" in the same way as in the case of 3 quasiperiods.

The asymptotic behavior of conductivity tensor $\sigma^{i k}$ is also the same in this case by the same reasons and the mean directions of the open trajectories (and the integer 4-tuples $\left.\left(m_{1}, m_{2}, m_{3}, m_{4}\right)\right)$ can be measured experimentally.

According to Novikov theorem the regions with "topologically regular" behavior can be found in any (arbitrarily small) open region of parameters $\boldsymbol{\eta}_{1}, \boldsymbol{\eta}_{2}, \boldsymbol{\eta}_{3}, \boldsymbol{\eta}_{4}, a_{1}$, $a_{2}, a_{3}, a_{4}, I_{1}, I_{2}, I_{3}, I_{4}$ and $B$. However, unlike the case $N=3$ there is no theorem here restricting the existence of "chaotic" trajectories only to the case of just one energy level $\left(\epsilon_{1}=\epsilon_{2}=\epsilon_{0}\right)$ containing open trajectories. As we also mentioned already, the case $N=4$ is much more complicated from topological point of view and there is no general classification of open trajectories in this case at the time. It is not clear also if the topologically regular behavior corresponds here to the generic situation or not and the probability to find the chaotic behavior is unknown for this situation.

Let us now make some more general remark about the Novikov problem in connection with $2 \mathrm{D}$ potentials $V(\mathbf{r})$. As can be seen, potentials $V(\mathbf{r})$ with rather many quasiperiods can be considered also as an interesting model of random potentials on the plane. This model is rather different from the standard models of random potentials but still can have common features with them for big $N$ when the chaotic behavior of the open trajectories appears. However, there is no strict theorems now which could connect Novikov problem with the problems of random potentials on the plane.

\section{CONCLUSION.}

We considered a special type of superlattices modulations giving quasiperiodic potentials $V(\mathbf{r})$ and $V_{B}^{\text {eff }}(\mathbf{r})$ on the plane. For this type of potentials we considered the "geometric limit" $(\tau \rightarrow \infty)$ of conductivity in the presence of magnetic field based on the global geometry of the level curves of $V_{B}^{e f f}(\mathbf{r})$. The main attention was paid to the so-called "topologically regular" behavior of non-singular open level curves for the cases of potentials with 3 and 4 quasiperiods. It was shown that it is possible to introduce "topological numbers" characterizing the asymptotic behavior of $\sigma^{i k}$ similar to the numbers introduced previously in the theory of normal metals. For the case of 3 quasiperiods it was possible to give also the description of structure of space of parameters giving potentials $V_{B}^{e f f}(\mathbf{r})$ according to the topological type of their non-singular open level curves. For the case of 4 quasiperiods only the part of the space of parameters corresponding to potentials close to "purely rational" was considered. It was shown that the corresponding "topological numbers" having the form of the integer 4-tuples can be also introduced in this case.

The author is grateful to Prof. S.P.Novikov for many fruitful discussions on this problem. The author is also grateful to Prof. I.A. Larkin who brought the articles on $2 \mathrm{D}$ electron gas to his attention for the interest to this work and advice.
* Electronic address: maltsev@itp.ac.ru

1 I.M.Lifshitz, M.Ya.Azbel, and M.I.Kaganov, Sov. Phys. JETP 4, 41 (1957).

2 I.M.Lifshitz and V.G.Peschansky, Sov. Phys. JETP 8, 875 (1959).

3 I.M.Lifshitz and V.G.Peschansky, Sov. Phys. JETP 11, 137 (1960).

4 I.M.Lifshitz and M.I.Kaganov, Sov. Phys. Usp. 2, 831 (1960).

5 I.M.Lifshitz and M.I.Kaganov, Sov. Phys. Usp. 5, 411 (1962).

6 S.P.Novikov, Russian Math. Surveys 37, 1 (1982).

7 A.V.Zorich, Russian Math. Surveys 39, 287 (1984).

8 S.P.Novikov, Proc. Steklov Inst. Math. 1 (1986).

9 S.P.Novikov, Proc. Conference "Topological Methods in Mathematics", dedicated to the 60th birthday of J.Milnor, June 15-22, S.U.N.Y. Stony Brook, 1991. Publish of Perish,
Houston, TX, pp. 223-233 (1993).

10 I.A.Dynnikov, Russian Math. Surveys 57, 172 (1992).

11 I.A.Dynnikov, Math. Notes 53, 495 (1993).

12 S.P.Tsarev, Private communication. (1992-93).

13 A.V.Zorich, Proc. Conf. "Geometric Study of Foliations" (Tokyo, November 1993) (T. Mizutani et al, ed.), World Scientific, pp. 479-498, Singapore 1994.

14 S.P.Novikov, Proc. Conf. of Geometry, December 15-26, 1993, Tel Aviv University (1995).

15 S.P.Novikov and A.Ya.Maltsev, JETP Lett. 63, 855 (1996).

16 I.A.Dynnikov, "Surfaces in 3-Torus: Geometry of plane sections." Proc.of ECM2, BuDA, 1996.

17 I.A.Dynnikov, Dissertation. Moscow State University of M.V.Lomonosov, Moscow (1996).

18 I.A.Dynnikov, "Semiclassical motion of the electron. A proof of the Novikov conjecture in general position and 
counterexamples." American Mathematical Society Translations, Series 2, Vol. 179, Advances in the Mathematical Sciences. Solitons, Geometry, and Topology: On the Crossroad. Editors: V.M.Buchstaber, S.P.Novikov, 45 (1997).

19 I.A.Dynnikov and A.Ya.Maltsev, JETP 85, 205 (1997).

20 A.Ya. Maltsev, JETP 85, 934 (1997).

21 S.P.Novikov and A.Ya.Maltsev, Physics-Uspekhi 41(3), 231 (1998).

22 I.A.Dynnikov, Russian Math. Surveys 54, 21 (1999).

${ }^{23}$ S.P. Novikov, Russian Math. Surveys 54, 1031 (1999).

24 S.P. Novikov, GAFA2000 - Visions in Mathematics. Towards 2000., Geom. func. anal. Special Volume, 406 (2000).

25 R.D.Leo, PhD Theses, University of Maryland. Department of Math., College Park, MD 20742, USA (2000).

26 A.Ya.Maltsev and S.P.Novikov, math-ph/0301033 (to be published in the Special Number of the Bulletin of the Braz. Math. Society (2003)).

27 D.Weiss, K.v. Klitzing, K. Ploog, and G. Weimann, Europhys. Lett., 8 (2), 179 (1989).

28 R.R. Gerhardts, D. Weiss, K.v. Klitzing, Phys. Rev. Lett. 62, 1173 (1989).

29 R.W. Winkler, J.P. Kotthaus, and K. Ploog, Phys. Rev. Lett. 62, 1177 (1989).

30 C. W. J. Beenakker, Phys. Rev. Lett. 62, 2020 (1989).

31 P. Vasilopoulos and F.M. Peeters, Phys. Rev. Lett. 63, 2120 (1989).

32 E.S.Alves, P.H. Beton, M. Henini, L. Eaves, P.C. Main, O.H. Hughes, G.A. Toombs, S.P. Beaumont, and C.D.W. Wilkinson, J. Phys. Condens. Matter 1, 8257 (1989).

${ }^{33}$ K. Ismail, D.A. Antoniadis, H.I. Smith, C.T. Liu, K. Nakamura, and D.C. Tsui, J. Vac. Sci. Technol. B 7, 2000 (1989).

${ }^{34}$ K. Ismail, T.P. Smith III, W.T. Masselink, and H.I. Smith, Appl. Phys. Lett. 55, 2766 (1989).

35 H. Fang and P.J. Stiles, Phys. Rev. B 41, 10171 (1990).

36 P. Středa and A.H. MacDonald, Phys. Rev. B 41, 11892 (1990).

37 A. Toriumi, K. Ismail, M. Burkhardt, D.A. Antoniadis, and H.I. Smith, Phys. Rev. B 41, 12346 (1990).

38 C.Zhang and R.R. Gerhardts, Phys. Rev. B 41, 12850 (1990).

39 F.M. Peeters and P. Vasilopoulos, Phys. Rev. B 42, 5899 (1990).

40 R.A. Puechner, J. Ma, R. Mezenner, W.-P. Liu, A.M. Kriman, G.N. Maracas, G, Bernstein, D.K. Ferry, P. Chu, H.H. Wieder, and P. Newman, Surf. Sci. 228, 520 (1990).

41 D. Weiss, K.v. Klitzing, K. Ploog, and G. Weimann, Surf. Sci. 229, 88 (1990).

${ }^{42}$ P.H. Beton, M.W. Dellow, P.C. Main, E.S. Alves, L. Eaves,
S.P. Beaumont, and C.D.W. Wilkinson, Phys. Rev. B 43, 9980 (1991).

43 D. Weiss, in Electronic Properties of Multilayers and Low Dimensional Semiconductor Structures, sdited by J.M. Chamberlain, L. Eaves. and J.-C. Portal (Plenum, New York, 1990), p. 25.

44 R.R. Gerhardts, D. Weiss, and U. Wulf, Phys. Rev. B 43, 5192 (1991).

45 R.R. Gerhardts, Phys. Rev. B 45, 3449 (1992).

46 F.M. Peeters and P. Vasilopoulos, Phys. Rev. B 46, 4667 (1992).

47 J.H. Davies and I.A. Larkin, Phys. Rev. B 49, 4800 (1994).

48 R. Taboryski, B. Brosh, M.Y. Simmons, D.A. Ritchie, C.J.B. Ford, and M.Pepper, Phys. Rev. B 51, 17243 (1995).

49 I.A. Larkin, J.H. Davies, A.R. Long, and R. Cusco, Phys. Rev. B 56, 15242 (1997).

50 J.H. Davies, D.E. Petticrew, and A.R. Long, Phys. Rev. B 58, 10789 (1998)

51 C. Albrecht, J.H. Smet, D. Weiss, K. v. Klitzing, R. Hennig, M. Langenbuch, M. Suhrke, U. Rössler, V. Umansky, and H. Schweizer, Phys. Rev. Lett. 83, 2234 (1999).

52 D.E. Grant, A.R. Long, and J.H. Davies, Phys. Rev. B 61, 13127 (2000).

53 J. Brüning, S.Yu. Dobrokhotov, and K.V. Pankrashkin, Russian Journ. of Math. Phys. 9:1, 14 (2002).

${ }^{54}$ I.V. Kukushkin, J.H. Smet, V.I. Falko, K. v. Klitzing, and K. Ebert, Phys. Rev. B 66, 121306-1 (2002).

55 A. Nogaret, Phys. Rev. B 66, 125302-1 (2002).

56 Let us use here the word "orbit" for the circular cyclotron electron orbit in the magnetic field B and "trajectory" for the drift of the center of cyclotron orbit in the presence of $V(\mathbf{r})$. We hope that there should be no misunderstanding because of two similar terms.

57 We are sorry for a little bit unnatural definitions of irrationality. We follow here the standard definitions arising from $3 D$ topology approach.

58 The last property was first mentioned in ${ }^{15}$ in the case of normal metals and called later the "Topological Resonance". This property plays an important role for the conductivity of normal metals making possible the experimental observation of "Topological Numbers" for this case.

59 The consideration $\mathrm{in}^{20}$ is based on the purely geometrical aspects and does not include the quantum corrections due to the jumps from one part of trajectory to another. It can be shown, however, that for chaotic trajectories of Dynnikov type these jumps should be also important for rather big values of $\tau$. 